\title{
The mass of our Galaxy from satellite proper motions in the Gaia era
}

\author{
T. K. Fritz ${ }^{1,2 \star}$, A. Di Cintio ${ }^{1,2} \dagger$, G. Battaglia ${ }^{1,2}$, C. Brook ${ }^{1,2}$, S. Taibi ${ }^{1,2}$ \\ ${ }^{1}$ Instituto de Astrofísica de Canarias, Calle Via Láctea s/n, E-38206 La Laguna, Tenerife, Spain \\ ${ }^{2}$ Universidad de La Laguna. Avda. Astrofísico Fco. Sánchez, La Laguna, Tenerife, Spain
}

Accepted XXX. Received YYY; in original form ZZZ

\begin{abstract}
We use Gaia DR2 systemic proper motions of 45 satellite galaxies to constrain the mass of the Milky Way using the scale free mass estimator of Watkins et al. (2010). We first determine the anisotropy parameter $\beta$, and the tracer satellites' radial density index $\gamma$ to be $\beta=-0.67_{-0.62}^{+0.45}$ and $\gamma=2.11 \pm 0.23$. When we exclude possible former satellites of the Large Magellanic Cloud, the anisotropy changes to $\beta=-0.21_{-0.51}^{+0.37}$. We find that the index of the Milky Way's gravitational potential $\alpha$, which is dependent on the mass itself, is the parameter with the largest impact on the mass determination. Via comparison with cosmological simulations of Milky Way-like galaxies, we carried out a detailed analysis of the estimation of the observational uncertainties and their impact on the mass estimator. We found that the mass estimator is biased when applied naively to the satellites of simulated Milky Way halos. Correcting for this bias, we obtain for our Galaxy a mass of $0.58_{-0.14}^{+0.15} \times 10^{12} \mathrm{M}_{\odot}$ within $64 \mathrm{kpc}$, as computed from the inner half of our observational sample, and $1.43_{-0.32}^{+0.35} \times 10^{12} \mathrm{M}_{\odot}$ within $273 \mathrm{kpc}$, from the full sample; this latter value extrapolates to a virial mass of $M_{\text {vir } \Delta=97}=1.51_{-0.40}^{+0.45} \times 10^{12} M_{\odot}$ corresponding to a virial radius of $R_{\text {vir }}=308 \pm 29 \mathrm{kpc}$. This value of the Milky Way mass lies in-between other mass estimates reported in the literature, from various different methods.
\end{abstract}

Key words: dark matter - Galaxy: fundamental parameters - Galaxy: halo - Galaxy: kinematics and dynamics - galaxies: dwarf

\section{INTRODUCTION}

In a $\Lambda$ CDM universe, galaxies are embedded in a dark matter halo (White \& Rees 1978), which is the most important component in terms of mass. The radial density of dark matter halos can be approximated by a NFW profile (Navarro et al. 1997), characterised by two parameters, e.g. its virial mass and concentration. Observationally, the virial mass of dark matter halos surrounding galaxies is difficult to obtain, since it is best measured at large distances from the centre of the galaxy itself, at radii where the number of dynamical tracers is low.

Having a good estimate of the MW virial mass would allow for more direct comparisons with cosmological simulations, specifically for those properties that are dependent on mass. For example, cosmological DM-only simulations have a number of satellites that have too much mass in the inner regions to be consistent with the observed internal kinematic

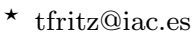

$\dagger$ Marie-Skłodowska-Curie Fellow properties of MW satellite galaxies . This issue, dubbed the 'Too big to fail' problem (Boylan-Kolchin et al. 2011), would be solved trivially if the mass of the MW was relatively low (Wang et al. 2012; Vera-Ciro et al. 2013).

Many methods employed so far for measuring the virial mass of the MW require extrapolation, because the dynamical tracers either do not extend out to the virial radius (e.g. globular clusters, see Harris (1996)), or their apparent magnitude at that distance makes observations with current facilities challenging (e.g. blue horizontal branch stars). The most easily accessible tracers that cover the largest radial range are dwarf galaxies. However, past attempts to use dwarf galaxies have resulted in a range of derived halo masses, which are particularly sensitive to which galaxies are included (especially Leo I Kulessa \& Lynden-Bell 1992; Watkins et al. 2010; Boylan-Kolchin et al. 2013): these measurements provide a total MW mass that ranges from 0.5 to $3 \times 10^{12} \mathrm{M}_{\odot}$ (see Wang et al. 2015a; Bland-Hawthorn \& Gerhard 2016, for reviews on the MW mass). A limitation of these previous studies was that systemic proper motions were only avail- 
able for a handful of MW satellite galaxies, with the consequence that the anisotropy parameter of the tracer population, $\beta$, could not be well constrained. The degeneracy between mass and anisotropy (see e.g. Binney \& Tremaine 2008) then leads to significant uncertainties in the mass estimation.

Recently, Gaia DR2 (Gaia Collaboration et al. 2018a) provided proper motions of more than 1 billion stars in our Galaxy and sub-systems within, such as globular clusters and satellites. These data have been used for quantitative determinations of the MW mass based on globular clusters (see e.g. Watkins et al. 2019, Vasiliev 2019) and fast halo stars (see e.g. Monari et al. 2018, Deason et al. 2019). Gaia DR2 systemic proper motions for more than 40 of the MW satellites have now been obtained (see e.g. Gaia Collaboration et al. 2018b, Fritz et al. 2018, Simon 2018, Pace \& Li 2019), the first such measurements for the faintest dwarfs. Callingham et al. (2019) considered the sample of classical dwarfs for a determination of the Milky Way mass, but for these systems previous HST based proper motions were already of similar precision (Patel et al. 2018).

In this work we use the Gaia DR2 proper motions of the full set of satellite dwarf galaxies to determine the virial mass of the Milky Way. For this purpose we adopt the Watkins et al. (2010) (Wa10) mass estimator, tailored to systems with full phase-space information. This mass estimator has the advantage of allowing arbitrary constant values for the anisotropy parameter, an arbitrary power-law mass profile for the underlying halo and an arbitrary powerlaw for the tracer distribution, without hidden assumptions on these quantities. Its applicability to the Milky Way has been previously tested using constrained cosmological simulations of the Local Group of galaxies (Di Cintio et al. 2012).

Other methods, like Jeans and Schwarzschild (Schwarzschild 1979) modelling, allow better constraints on the mass profile, but they contain a significant number of free parameters, making them difficult to apply to a still relatively small sample. Further, the reliance on simulations is minimal in our methodology, less than when simulations are directly used, as in Patel et al. (2018) or Callingham et al. (2019).

The paper is structured as follows: in Section 2 we describe the data and sample used; in Section 3 we derive the parameters used in the Wa10 mass estimator; in Section 4 we compare with simulations to assess the biases of the estimator. An additional complication is given by the presence of a close-by and relatively massive neighbour, the Large Magellanic Cloud (LMC), which exerts a strong gravitational pull (Gómez et al. 2015; Garavito-Camargo et al. 2019). In Section 5 we present our assumptions regarding the reflex motion of the MW due to the Large Magellanic Cloud (LMC) and explore the effect of galaxies in the sample that are possible former satellites of the LMC. In Section 6 we derive the mass (profile) of the Milky Way and compare it with other determinations. Finally, in Section 7, we conclude and summarise our results.

\section{DATA}

Our sample consists of all satellite galaxies within the virial radius of the MW for which systemic proper motions and line of sight velocities are available, which minimises the dependence on the anisotropy parameter, $\beta$. We include satellites galaxies from Fritz et al. (2019), Carlin \& Sand (2018), Gaia Collaboration et al. (2018b), Torrealba et al. (2019), and Longeard et al. (2019). Details of the sources of proper motions, line-of-sight velocities and distance moduli are listed in Appendix A. We exclude Laevens 1/Crater I, as it is likely a globular cluster (see Laevens et al. 2014, Kirby et al. 2015, Voggel et al. 2016, Weisz et al. 2016). We also exclude Phoenix I and Eridanus II because their large distances imply that they are likely outside the virial radius of the MW.

The proper motions are primarily based on Fritz et al. (2018), which relied on samples of individual stars for which spectroscopic measurements were available. This reduces the likelihood that a proper motion measurement is affected by membership uncertainties. We assume systematic uncertainties of 0.035 mas/yr (Gaia Collaboration et al. 2018b) in the proper motion for Bootes III, Antlia II and Sagr II since these were not included in the original sources.

In total, our sample contains 45 satellite galaxies, as shown in Fig. 1. In the following, we also consider subsamples of these 45 galaxies based on the uncertainty in the amplitude of the velocity vector, and on the possible origin as a former satellites of the LMC. These sub-samples will be defined in detail in the appropriate sections but Fig. 1 can be used for an overview. ${ }^{1}$

To test for the radial mass distribution and consistency of our results, we also divide the sample into two bins: an inner one, which includes satellites within $64 \mathrm{kpc}$ (with the outermost object being Bootes I), and an outer one with satellites found beyond $77 \mathrm{kpc}$ (with the innermost one being Ursa Minor). With such division we achieve roughly equal sample sizes and also ensure that the inner and outermost galaxies are independent of LMC satellite selection.

\section{DETERMINATION OF $\alpha, \beta$ AND $\gamma$ PARAMETERS}

When galactocentric distances and total velocities are available for a sample of tracers, the Wa10 mass estimator gives the total mass of a galaxy within the radius of its outermost tracer with the equation:

$M_{<=\text {out }}=\frac{1}{G} \frac{\alpha+\gamma-2 \beta}{3-2 \beta} r_{\mathrm{GC} \text { out }}^{1-\alpha}\left\langle v_{\mathrm{tot}}^{2} r_{\mathrm{GC}}^{\alpha}\right\rangle$

where $r_{\mathrm{GC}}$ is the Galactocentric radius of the tracer $\left(r_{\mathrm{GC}}\right.$ out being the radius of the outer most one), $v_{\text {tot }}$ is the total velocity of the tracers and $\alpha, \beta$ and $\gamma$ are, respectively, the parameters describing the radial mass distribution of the host galaxy, the anisotropy parameter (assumed to be independent of radius) and the radial distribution of the tracer population (here the MW satellite galaxies). The estimator assumes that the tracer population has a number density that follows a power-law and moves in a scale-free potential.

1 In principle, a few objects could have been satellites of the Small Magellanic Cloud (SMC), but since the SMC was itself very likely a satellite of the LMC, we do not distinguish between the two cases. 


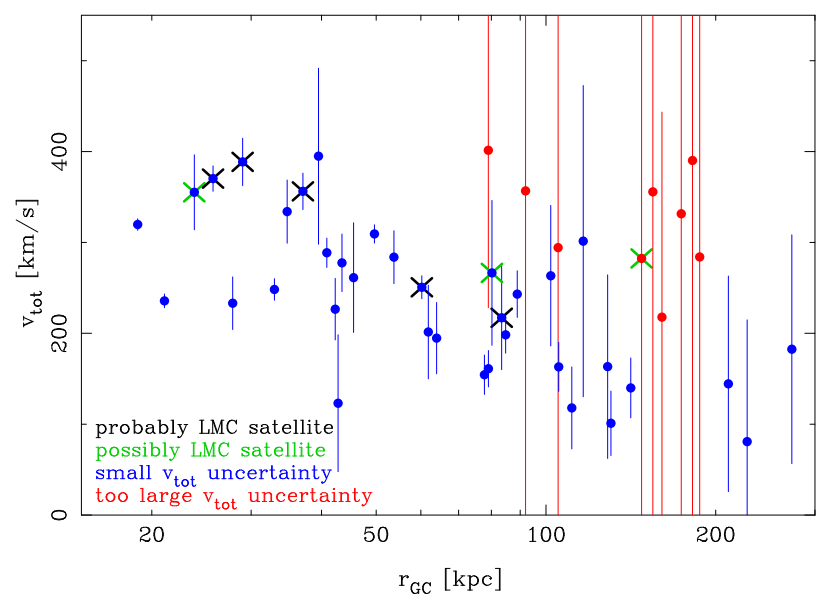

Figure 1. Total velocity (i.e. amplitude of the velocity vector) versus Galactocentric distance for the sample of 45 galaxies. In red we indicate those galaxies that we exclude from the final sample used for the mass determination, due to the very large uncertainty in their total velocity (see Section 4.2 and Fig. 8 for details). The crosses show the galaxies that are likely to be former LMC satellites.

We derive the parameters $\alpha, \beta$ and $\gamma$ for the MW in the following sections. In addition, we also calculate their values in simulations for comparison and in order to correct for biases in the estimates of the MW mass.

\subsection{Determination of the mass index $\alpha$}

The mass profile can be written as:

$$
M(r) \propto r_{\mathrm{GC}}^{1-\alpha}
$$

where $\alpha$ can be obtained by fitting the full observational data set of tracers at every radii, or by simply interpolating between the inner most and the outer most point of the mass distribution. Given the assumption of a power-law, we decided to use this last method $^{2}$ : we therefore need to calculate the mass enclosed within the innermost and outermost radius of the objects in our sample.

Estimates of the MW mass within the inner radius of our tracer sample $(\approx 20 \mathrm{kpc})$ agree relatively well in the literature. For example, Küpper et al. (2015) obtained $\mathrm{V}_{\text {circ }}=217_{-19}^{+22} \mathrm{~km} / \mathrm{s}$ at $\approx 19 \mathrm{kpc}$ using the Pal 5 stream; Bovy et al. (2016) obtained $\mathrm{V}_{\text {circ }}=198 \pm 9 \mathrm{~km} / \mathrm{s}$ at $20 \mathrm{kpc}$ using the Pal 5 and GD1 streams; Watkins et al. (2019) obtained $\mathrm{V}_{\text {circ }}=213_{-17}^{+20} \mathrm{~km} / \mathrm{s}$ at $21.1 \mathrm{kpc}$ using globular cluster dynamics. We average these measurements and obtain $\mathrm{V}_{\text {circ }}=215 \pm 20 \mathrm{~km} / \mathrm{s}$, and we then convert such circular velocity to a mass at $19 \mathrm{kpc}$. We then calculate $\alpha$ as a function of different assumed values of the mass within the outermost point $\left(r_{\mathrm{GC}}=273 \mathrm{kpc}\right)$, keeping the value of the mass within $19 \mathrm{kpc}$ fixed. The results are shown in the top panel of Fig. 2 as red points with error-bars: the variation of $\alpha$ with outer mass is well fit by the quadratic function ${ }^{3}$ :

$$
\alpha=11.69-1.016 \times \log M_{273}+0.0063 \times\left(\log M_{273}\right)^{2}
$$

2 We nevertheless verified, using the simulations, that the two methods provide the same value of $\alpha$ within 0.02 .

3 As elsewhere in this paper log is in base 10 .
We estimate an uncertainty in $\alpha$ of 0.07 , taking into account the uncertainty of the mass at $19 \mathrm{kpc}$. Eq. 2 will be later used in Section 6 to iteratively calculate $\alpha$ and its associated mass value. Following a similar procedure we also calculate $\alpha$ as a function of mass for two other radial ranges, respectively the inner (between 19 and $64 \mathrm{kpc}$ ) and outer (between 77 and $273 \mathrm{kpc}$ ) range. The results are shown respectively in the central and bottom panels of Fig. 2, as red points with error-bars. Once again, a quadratic relation is used to compute the variation of $\alpha$ with mass: for the inner range, it reads as $\alpha=20.43-1.554 \times \log M_{64}-0.0146 \times$ $\left(\log M_{64}\right)^{2}$ with an uncertainty on $\alpha$ of 0.159 , while for the outer range we get $\alpha=11.66-1.001 \times \log M_{273}+0.0057 \times$ $\left(\log M_{273}\right)^{2}$ with an uncertainty of 0.033 .

We now proceed to compute the $\alpha$ parameter using both hydrodynamical as well as dark matter only simulations. For this purpose, we use the MaGICC (Brook et al. 2012) and NIHAO (Wang et al. 2015b) hydrodynamic simulations, selecting Milky Way-like galaxies that have a similar circular velocity as the one of our Galaxy: we found that when $V_{\text {circ }}$ at $19 \mathrm{kpc}$ is consistent with observations, the obtained value of $\alpha$ agrees well between simulations and observations (NI$\mathrm{HAO} / \mathrm{MaGICC}$ shown as green points in Fig. 2).

For a more statistically significant sample, we used the ELVIS dark matter only (DMO) simulation set (Garrison-Kimmel et al. 2014), which, being simulations of the local group of galaxies, have the advantage that the main halos have a similar environment as the MW, i.e. there is a M31 sized halo at about the correct distance from the MW and no other massive halo within $2.8 \mathrm{Mpc}$. We selected all MW and M31 halos, for a total of 24 objects, and retrieved the masses at the bins borders $(19,64,77$ and $273 \mathrm{kpc})$ which allowed us to again compute $\alpha$ by interpolating between inner-most and outer-most mass using a power-law. We compared these results with the $\alpha$ obtained from hydrodynamical simulations and from our observations in Fig. 2, where the 24 ELVIS points are cyan. As expected, the value of $\alpha$ in the DMO case is lower than in the hydrodynamical simulations and in the observed case, since baryons cool towards the centre of the halo making the profile more concentrated for $\mathrm{r} \rightarrow 0$.

In summary, in the mass range probed by the hydrodynamical simulations $\left(\sim 1.2 \times 10^{12} \mathrm{M}_{\odot}\right)$ the average values of $\alpha$ derived from both simulations and MW observations fall in the range $\alpha \sim 0.3-0.4$.

\subsection{Determination of the anisotropy parameter $\beta$}

The velocity anisotropy is defined as

$\beta=1-\left(\sigma_{\theta}^{2}+\sigma_{\phi}^{2}\right) /\left(2 \sigma_{\mathrm{r}}^{2}\right)$

where the terms are the velocity dispersions in spherical coordinates relative to the Galactic centre. Given the assumption that the velocity anisotropy is independent of radius for the Wa10 estimator, we first derive $\beta$ for the full sample.

In order to calculate the velocity dispersion in the radial and tangential directions, we first transform the observed heliocentric systemic line-of-sight velocities and proper motions of each galaxy into velocities in a Galactocentric system; the associated uncertainties are estimated by Monte Carlo simulations, considering the uncertainties (statistical and systematic) in 

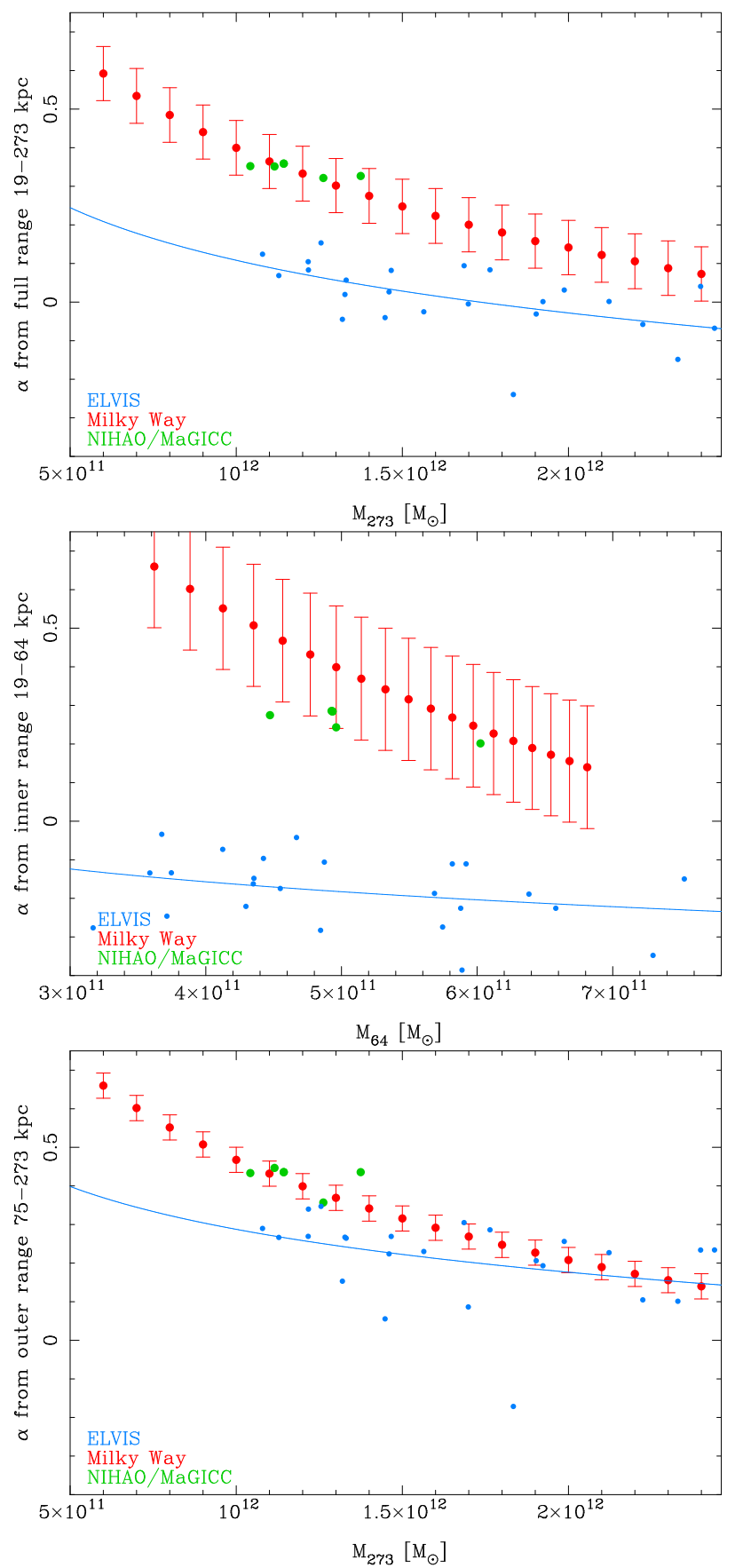

Figure 2. $\alpha$ as function of assumed mass at different outer radii (red circles with error-bars). We also show $\alpha$ obtained in hydrodynamical NIHAO/MaGICC simulations, in green, using only those simulated galaxies that have a $\mathrm{v}_{\text {circ }}$ at $19 \mathrm{kpc}$ within $215 \pm 20 \mathrm{~km} / \mathrm{s}$, and in dark matter only (ELVIS) simulations, as blue circles, where the solid line is a fit to the points. From top to bottom, the panels show results for the full radial range $(19-273 \mathrm{kpc})$, and the inner (19-64 kpc) and outer (77-273 kpc) sample.

proper motions, line of sight velocities, distance of the galaxy, and distance $\left(\mathrm{R}_{0}\right)$ (Bland-Hawthorn \& Gerhard 2016; Gravity Collaboration et al. 2019) and velocity of the Sun (Reid \& Brunthaler 2004; Schönrich et al. 2010) relative to the Galactic centre. Here, the most important contribution by far comes from the proper motion uncertain- ties. The average and standard deviation of the velocities obtained from the Monte Carlo simulations are then used as the value and uncertainty of each galaxy's velocity.

For obtaining velocity dispersions, and therefore $\beta$, care is needed to ensure that large proper motion uncertainties are not interpreted as enhanced tangential dispersion, which would bias $\beta$ towards more negative values. To this aim, we use a Bayesian approach very similar to that by Fritz et al. (2019). We run the MultiNest code (Feroz et al. 2009; Buchner et al. 2014), which is a multimodal nested sampling algorithm, to perform the posterior parameter estimation. We assume that the velocity distributions are Gaussian, taking into account measurement uncertainties on the individual quantities to determine the intrinsic spreads. We treat each component of the velocity dispersion separately and fix the average velocity to zero. We adopted flat priors for the velocity dispersion components between 0 and 500 $\mathrm{km} / \mathrm{s}$. The dispersions are determined well enough that we do not expect the choice of the prior to have a significant influence.

The distribution of velocity anisotropies is derived by applying Eq. 3 to random draws of values from the posterior distribution of the velocity dispersion in the radial, azimuthal and polar direction.

Fig. 3 shows the $\beta$ obtained for the different samples of tracers being considered. The full sample of 45 satellites, whose posterior distribution is shown as solid black line in Fig. 3, yields an anisotropy $\beta=-0.92_{-0.65}^{+0.49} 4$ very similar to the determination by Riley et al. (2019) $\left(\beta=-1.05_{-0.49}^{+0.39}\right)$, which is not surprising since our sample has only 7 more galaxies compared to theirs ${ }^{5}$. Allowing the mean velocities to be different from zero, $\beta$ changes slightly to $\beta=-0.61_{-0.54}^{+0.39}$, although its value is fully compatible with the previous determination within $1 \sigma^{6}$.

If we restrict the sample to the 36 satellite galaxies with the smallest uncertainties on the total velocity (the sample shown in blue in Fig. 1), the velocity anisotropy becomes $\beta=-0.67_{-0.62}^{+0.45}$, still consistent with the value from the full sample, but less tangential. This case is represented by a red posterior distribution in Fig. 3.

It is likely that the assumption of a velocity anisotropy independent of radius made in the Wa10 mass estimator is violated in reality, as e.g. indicated by the data (Riley et al. 2019) and as expected due to tidal disruption of satellites at small distances (Kelley et al. 2019; Samuel et al. 2019). Since this could have an impact on the inferred mass, here we test for such radial variations of $\beta$ (see also Section 4 ), by considering separately the objects belonging to the inner and outer samples defined in Section 2. The inner sample yields

4 The uncertainties represent the $68.3 \%$ confidence interval around the median all through the paper.

5 On the other hand, our uncertainties on the velocity anisotropy are larger than theirs, and we suspect that it is because we do not assume $\sigma_{\theta}=\sigma_{\phi}$; indeed the measurements from our sample suggest that they are significantly different from each other $\left(\sigma_{\phi}=\right.$ $101 \pm 13 \mathrm{~km} / \mathrm{s}$ and $\left.\sigma_{\theta}=192 \pm 24 \mathrm{~km} / \mathrm{s}\right)$.

6 While we obtain an average radial and azimuthal velocity consistent with zero, we find $\mathrm{v}_{\boldsymbol{\theta}}=-86 \pm 28 \mathrm{~km} / \mathrm{s}$, which is not unexpected because it is known that the majority of galaxies rotate in one direction within the vast polar structure (Pawlowski et al. 2012; Fritz et al. 2018). 


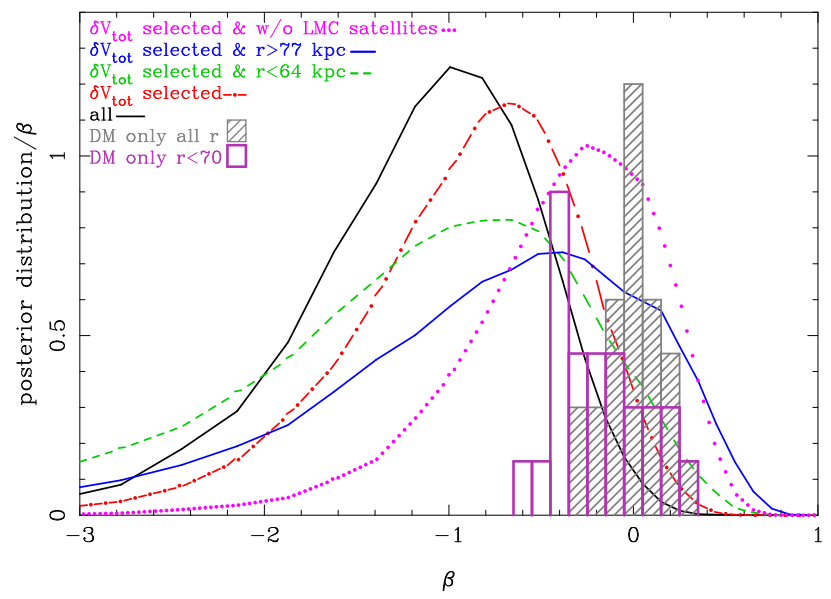

Figure 3. $\beta$ anisotropy parameter probability distributions, for different samples of satellites. For the case excluding likely former LMC satellites we only show the most extreme case, where all are excluded. Plotted is the binned probability scaled by the width of the bin. We also show the most likely $\beta$ from $24 \mathrm{DM}$ only simulations from the ELVIS suite, both for all subhalos within $280 \mathrm{kpc}$, and once only the ones within $70 \mathrm{kpc}$ are used. These two distributions are scaled such that they fit on the plot.

$\beta=-0.76_{-0.96}^{+0.63}$ (shown as a green distribution in Fig. 3), while for the outer one we get $\beta=-0.41_{-0.90}^{+0.56}$ (shown in blue in Fig. 3). While this might hint to a slight increase in the value of this parameter as a function of radius, the uncertainties are at present too large to pin this down. In any case, such a small difference in the values of $\beta$ would cause a negligible variation in the mass determination with the Wa10 estimator (see Fig. 1, left panel, for the $\gamma=2$ case in Di Cintio et al. 2012).

We also investigate whether the exclusion of the most likely, as well as tentative, former LMC satellites does have an effect on $\beta$, finding that the value becomes closer to isotropic $\left(\beta=-0.32_{-0.53}^{+0.39}\right.$ in the former case and $\beta=$ $-0.21_{-0.51}^{+0.37}$ in the latter case, see also Fig. 3). This is somewhat expected, given the large tangential velocities of the satellites possibly associated to the LMC. Nonetheless, this variation is not expected to have a significant impact on the MW mass determination (Di Cintio et al. 2012).

Since we will use cosmological simulations to address possible biases in the MW mass estimate (see Section 4) here we also compute the anisotropy parameter for the ELVIS simulations. Considering all the subhalos within $280 \mathrm{kpc}$ from the Galactic centre, we obtain an average of $\beta=0.00$ with a scatter of 0.15 over all simulations (shown as grey histogram in Fig. 3), while when selecting only satellites within $\mathrm{r}<70 \mathrm{kpc}, \beta$ is -0.18 (purple histogram in Fig. 3).

Note that we have not included the determination of $\beta$ from hydrodynamical simulations since we found that they contain a lower number of luminous sub-haloes, particularly in the inner regions, compared to observations, which makes the calculation less meaningful. This also applies to the calculation of $\gamma$ in the next section.

\subsection{Determination of tracer power-law index $\gamma$}

The parameter $\gamma$ is the power-law index of the tracer number density distribution. When considering the cumulative radial
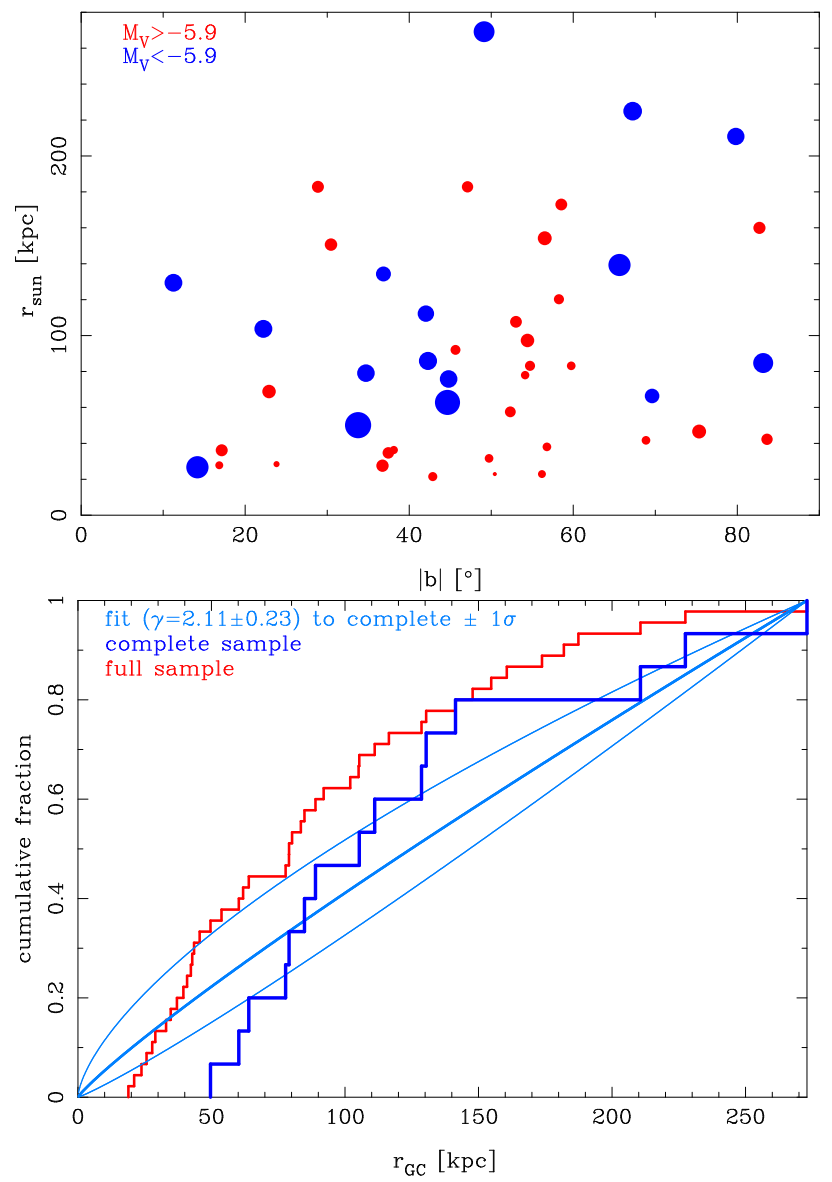

Figure 4. Top panel: Heliocentric distance of the satellite galaxies against the absolute value of their galactic latitude. Galaxies in the complete luminosity range (Koposov et al. 2008) at high latitudes are coloured in blue, while the full sample is shown in red. The size of the circles is larger for brighter galaxies. Bottom panel: Cumulative distribution of the full and of the complete sample, with corresponding fit to the latter, shown as cyan lines with $1 \sigma$ interval.

distribution of the satellites, $N_{\text {cum }}$, this follows a power-law of index $3-\gamma$.

The main difficulty in the determination of $\gamma$ originates in its dependence on the completeness of the galaxy surveys from which the satellites were detected. Koposov et al. (2008) calculated that at $260 \mathrm{kpc}$, approximately the distance of Leo I, SDSS (York et al. 2000) is complete down to an absolute magnitude $\mathrm{M}_{V}=-5.9$. ATLAS (Shanks et al. 2015), Pan-STARRS-1 (Chambers et al. 2016) $(\delta>-30)$, DES (Abbott et al. 2018) and some other smaller programs (Torrealba et al. 2018; Koposov et al. 2018; Nidever et al. 2017) have surveyed most of the remaining sky to similar or higher depths (Jethwa et al. 2016). Hence we make the assumption that these surveys are all complete at least to an absolute magnitude $\mathrm{M}_{V}=-5.9$ (see recent quantification of the completeness of PS1 by Drlica-Wagner et al. (2019)).

Already Pan-STARRS-1 and the NOAO all sky catalogue cover together nearly the full sky besides a few small regions. In practice, the main problem is not that some regions are missing completely, but that these surveys are less effective in detecting satellites closer to the Galactic plane due to the greater density of Galactic stars and extinction. 
To get a feeling of how poor the completeness is close to the Galactic plane, especially for the sample complete in luminosity, in Fig. 4 we plot the heliocentric distance against the absolute Galactic latitude $(|b|)$ of the satellites in our sample, where the size of the circles is larger for brighter galaxies. The magnitude-complete sample, made of all galaxies brighter than $\mathrm{M}_{V}=-5.9$, is indicated in blue, while the full sample is shown in red.

First, we notice that the lack of any galaxies within $|b|=11$ degrees is probably caused by incompleteness, but it is only marginally relevant for the sample's radial distribution: since all satellites are at a distance from the Galactic Centre (GC) that is more than double the distance between the GC and the sun, galaxies at all distances are nearly equally missed due to the zone of avoidance. Second, the galaxy closest to the plane is Antlia II (Torrealba et al. 2019): with $\mathrm{M}_{V}=-9.0$ and a distance of $130 \mathrm{kpc}$ it is fainter and more distant than the median galaxy in the complete sample, and is at a fairly average Galactic longitude $\left(l=-95^{\circ}\right)$. It is therefore unlikely that satellites with less extreme properties are missing within this distance.

In contrast, the galaxy with the second smallest $|\mathrm{b}|$ is Sagr I, the closest and one of the brightest satellites with $\mathrm{M}_{V}=-13.5$ (the median luminosity is $\mathrm{M}_{V}=-9.2$ in the complete sample): with $l=5.6^{\circ}$ it is in a region where galaxies are particularly difficult to discover due to the bulge. We therefore assume that a more distant and fainter galaxy would not have been discovered at the location of Sagr I, and so we exclude Sagr I from the main sample, while we keep Antlia II. The next galaxy brighter than the completeness limit of Koposov et al. (2008) is Carina I at $|b|=22^{\circ}$ : since it is about at the median distance and luminosity of the complete sample we include it. At larger $|\mathrm{b}|$ than Carina I there is likely no bias, since many fainter galaxies have been discovered there.

We are left with a total of 15 galaxies brighter than $\mathrm{M}_{V}=-5.9$, which constitutes our magnitude-complete sample, indicated as blue circles in Fig. 4, top panel.

We now proceed to fit a single scale free power-law to the radial distribution of our full and magnitude-complete satellite samples ${ }^{7}$, starting from a radius of $\mathrm{r}_{\mathrm{GC}}=0$. The fit is shown in cyan in the bottom panel of Fig. 4 for the magnitude complete sample: we obtain our main index of $\gamma=2.11 \pm 0.23$. We use Monte Carlo simulations to obtain the uncertainty, and we also check whether distance uncertainties matter, finding that they are irrelevant compared to Poisson uncertainties. We also fit the same sample without the SMC, since that galaxy is likely not independent of the LMC (see Section 5) and obtain $\gamma=2.08 \pm 0.25$. When we include all galaxies, $\gamma$ only increases slightly to $2.24 \pm 0.12$, meaning that an index larger than 2.24 seems unlikely.

Given the minor differences from the various cases, for the mass estimator we use the $\gamma$ index and uncertainty derived from the magnitude complete sample $(2.11 \pm 0.23)$.

For comparison, and for the assessment of biases in the

7 We note, however, that in reality there is a dearth of satellite galaxies within $18 \mathrm{kpc}$ from the Milky Way's centre: in our full sample the number of galaxies per radial interval is approximately constant, with a value of 0.5 galaxies per kpc between 18 and 48 kpc; however, we observe no galaxy within $18 \mathrm{kpc}$. Thus, the single power-law as defined in Wa10 must be used with caution.

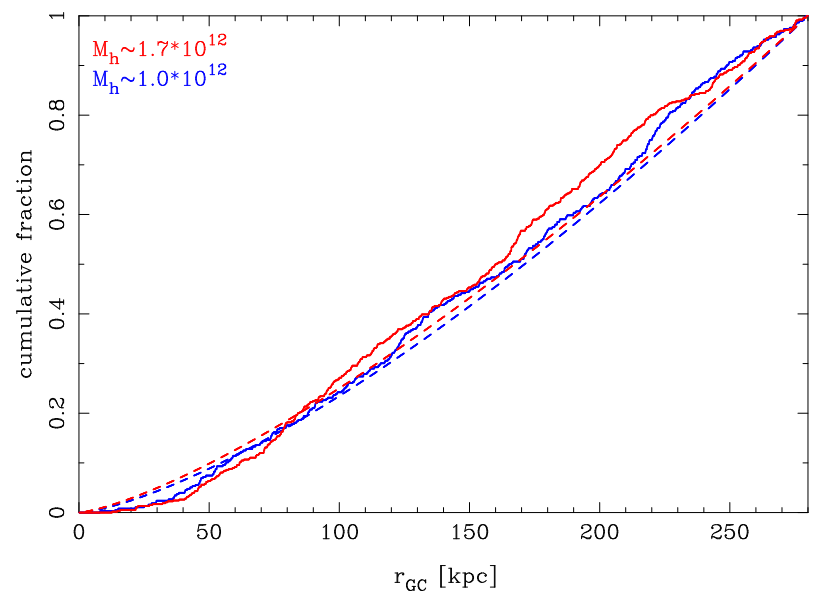

Figure 5. Cumulative number density profile with power-law fits for 2 typical ELVIS halos (in blue and red, with fits as dashed lines). A single power-law is not a good fit to the central region of the subhalo distribution.

mass determination (see Section 4), we calculate the $\gamma$ index of the ELVIS simulations in the same way, using all subhalos within $273 \mathrm{kpc}$. We obtain $\gamma=1.60 \pm 0.09$ where the uncertainty comes from the scatter amongst the different simulated MW-halos. The slope is about $2 \sigma$ smaller than the measurement inferred for the MW. We also note that a power-law distribution does not appear to be a perfect representation of the radial distribution of sub-haloes in the ELVIS simulations: as it can be appreciated in Fig. 5 for two examples, a power-law over-predicts the counts at small $\mathrm{r}$ and under-predicts them at intermediate and large radii. However, since the simulation uses only dark matter it is also not necessarily expected that they reproduce observations fully, due to effects like additional tidal destruction by the baryonic disk (see e.g. D'Onghia et al. 2010, Garrison-Kimmel et al. 2018, Riley et al. 2019, Kelley et al. 2019).

\section{ASSESSMENT OF BIASES}

We use cosmological simulations to test whether issues such as selection effects and observational uncertainties might bias the mass estimates. For this, we focus on the ELVIS suite because of their statistical significance, since this sample consists of 24 Milky-Way-like halos, with a virial mass between 1.0 and $2.8 \times 10^{12} \mathrm{M}_{\odot}$.

We select all resolved subhalos within $280 \mathrm{kpc}$, without requiring them to be bound, although in practice nearly all of them are (Boylan-Kolchin et al. 2013). The simulations contain between 324 and 813 (average of 539) subhalos.

From each simulation we select a sample of 45 subhalos as close as possible in Galactocentric distance to our sample of galaxies. Overall the simulations contain enough subhalos to find a good match for all the observed galaxies, with the median difference in Galactocentric distance between best match and the corresponding observed galaxy of $0.2 \mathrm{kpc}$. The match is slightly worse at small radii $(r<30 \mathrm{kpc})$, where the median difference in Galactocentric distance between best match and the corresponding observed galaxy is $1.4 \mathrm{kpc}$. The difference is noticeably larger for the subhalos 
of less massive hosts, reaching a median of $9 \mathrm{kpc}$ at $r<30$ $\mathrm{kpc}$ in the worst case, due to the lower number of subhalos.

To ensure that distance differences between simulations and observations do not matter (for example due to distance dependent tangential velocity uncertainties), we always adopt the values of the distance of the observed galaxies. We also adjust the position on the sky to the 'observed position', to preserve the influences of LOS velocities on the tangential velocities and other properties. However, we do not modify the velocity in spherical coordinates, so as to preserve the velocity anisotropy $\beta$. Since the rotation curve of the Milky Way is relatively flat in this radial range (Iocco et al. 2015), the impact of not adapting the velocities to the new distance is small. We checked for it and find that the mass measured with the estimator changed at most by $0.9 \%$ (on average by $0.1 \%$ ) for the full sample, and at most by $4 \%$ (on average by $0.5 \%$ ) for the sample within $64 \mathrm{kpc}$ compared to the mass obtained using the original radii.

We use $\gamma$ and $\beta$ as determined from the full sample of sub-haloes, whose values have been presented in Sections 3.2 and 3.3. In this way we can estimate the impact of the selection as independently as possible from the impact of uncertainties in $\beta$ and $\gamma$. For the mass index $\alpha$, we use relations between $\alpha$ and mass as derived in Section 3.1.

\subsection{Observational uncertainty-free case}

We first determine the correction factor of the Wa10 mass estimator when applied to simulations without including observational uncertainties. For each of the three sampled regions (full, inner and outer radial range) we start with an initial value of $\alpha$ by using the average of all simulations, $\alpha=0.02$. We then iterate: we use the initial $\alpha$ in the first iteration to determine the mass and its correction factor (i.e. $f_{\text {cor }}$, the ratio between the estimated mass, mass est, and the true mass, masstrue), and then use the previously derived mass dependency of $\alpha$ (Eq. 2 for the full range), to determine $\alpha$ for the second iteration. We then use this result for each mass to determine a new relation. A $3^{d}$ and $4^{\text {th }}$ iteration follow. By the $4^{\text {th }}$ iteration the change in the correction factor is less than $0.1 \%$.

We show in Fig. 6 the ratio of estimated to known mass against the estimated mass for the different samples. There is a clear offset, which is slightly larger for larger masses, particularly when the full sample is used.

We calculate the significance of the trend, rescaling such that the reduced $\chi^{2}=1$ : it follows that the trend has a significance of $3.4 \sigma$ for the full sample. On average, the correction factor is 1.23 for the full sample, 1.33 for the inner sample and 0.79 for the outer sample. Dividing the typical uncertainty through the correction factor gives a typical relative mass uncertainty of 0.16 for the full sample.

We tested whether these biases are peculiar of the selected samples by repeating the analysis on samples of tracers in which the parameters $(\alpha, \beta, \gamma)$ fulfil exactly the assumptions and definitions of the Wa10. These mock data-sets were constructed from distribution functions using Monte Carlo simulations ${ }^{8}$. We then select from these

8 The mock data were kindly provided by Laura Watkins (private communication).

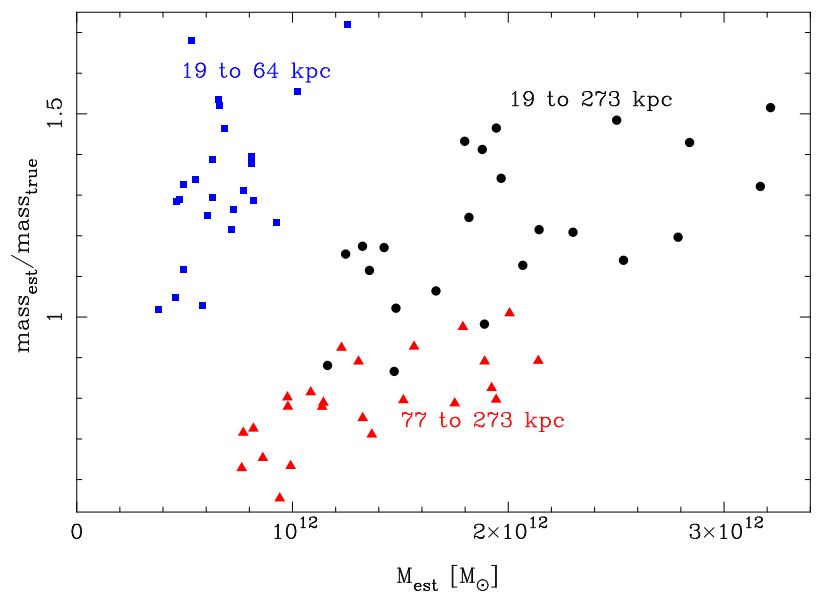

Figure 6. Test for biases in the Wa10 mass estimator: ratio of mass estimated to true mass in the uncertainty free case for the dark matter only ELVIS cosmological simulations. The subhalos are selected such that they match the radial distribution of the MW satellites used. The results from the full sample are indicated in black, while for the inner and outer sample in blue and red, respectively. Each symbol represents one MW-simulated galaxy in the ELVIS suite.

mock data 'subhalos' at the same distances as the galaxies observed, and find that the mass obtained does not suffer from any bias. Therefore we are reassured that the mass estimator works in the ideal case, thus the reason for the bias must be that the simulations do not fulfil all assumptions of the mass estimator.

While overall the masses are overestimated, the mass is underestimated in the outer sample. We suspect that the reason is that in the outer sample the majority of the subhalos are close to their apocenters. In contrast, in the inner part the majority are close to their pericenter, causing the opposite bias. The full sample is in between but also overestimates the mass, because increasing incompleteness with radius means that the subhalos are more likely close-in, and thus close to their pericenters.

\subsection{Adding observational uncertainties}

Observational uncertainties in the systemic proper motions typically bias velocities towards more tangential values, see e.g. Fritz et al. (2018). Since the mass estimator requires the same weighting for each galaxy, we cannot estimate the bias corrected measurement accounting for measurement uncertainties as in, for example, a Bayesian approach. Therefore, we apply forward modelling on the simulations and then estimate the correction factor as in Section 4.1.

In practice, we only consider the effect of uncertainties in the proper motion estimates, as the other sources of error are negligible in comparison. This is implemented by extracting new proper motion values from a Gaussian distribution centred on the 'observed' proper motions of the sub-haloes in the simulations and with dispersion equal to the proper motion uncertainty. The resulting properties are then propagated to the total velocities. For each simulated MW-analogue we perform 2000 independent draws, and calculate the average mass (and scatter) iteratively.

As with the uncertainty free case, we obtain the correc- 

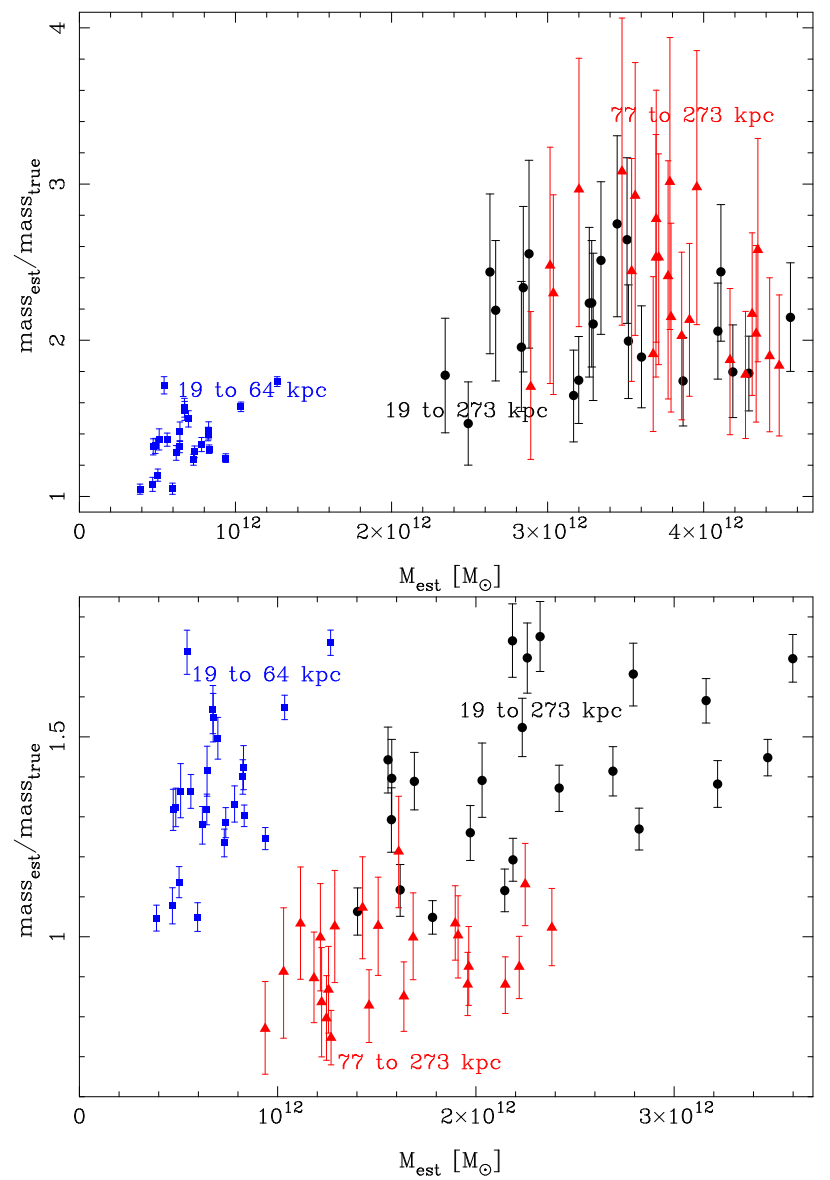

Figure 7. Ratio of mass estimator to true mass in the dark matter only ELVIS simulations, with observational uncertainties added. Here, error-bars enclose the $1 \sigma$ intervals as obtained from $68.3 \%$ confidence intervals. The subhalos are selected to match the radial distribution of the sample of MW satellites used in this analysis. This sample is then divided into an inner (blue), outer (red) and full (black) sample. Top panel: results for the whole sample. Bottom panel: we omit galaxies whose inclusion results in a loss of precision in the results, due to the large uncertainties in the velocity determination (see text). None of those is within $64 \mathrm{kpc}$, thus these points are identical in the two panels.

tion factor as a function of the estimated mass (see Fig. 7). The obtained data are then fit as a linear function of $m_{\mathrm{est}}$, where we weight all simulations equally. We also fit the scatter between the different trials as a linear function of $m_{\text {est }}$ with equal weights to obtain the typical uncertainty.

As shown in Fig. 7 (top panel) the correction factor for the full sample increases from the uncertainty free case of 1.23 to 2.1 . Dividing the uncertainty by the correction factor we obtain a typical relative uncertainty of 0.2 on the mass. When the scatter between the different simulations is added, the total relative mass uncertainty is 0.26 (to be compared to the value of 0.16 for the uncertainty free case).

Can the exclusion of some objects reduce the relative mass uncertainty? To identify which objects might produce a large uncertainty in the mass estimate, we use the sample created from the simulations to obtain the relative mass uncertainty of each subhalo. Using the galaxy dependent part

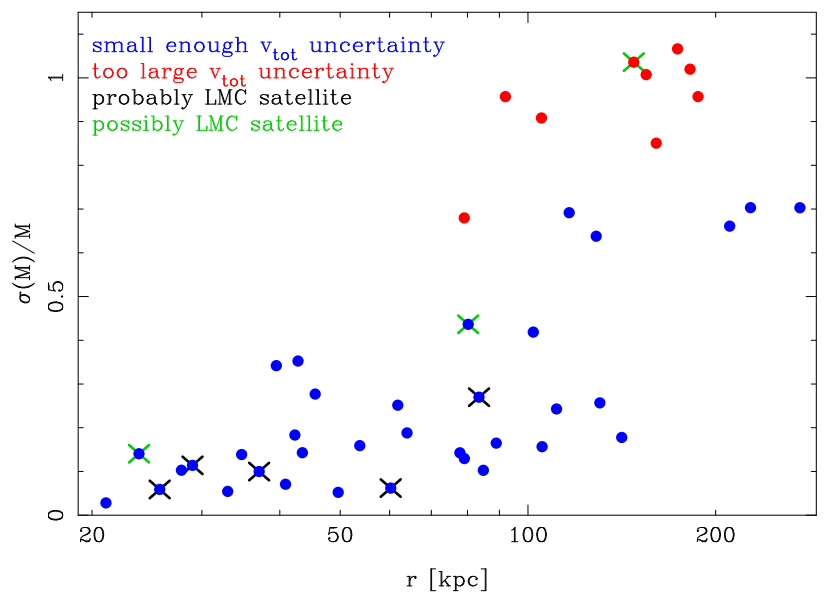

Figure 8. Relative mass uncertainty due to proper motion uncertainties for each simulated subhalo matched to an observed satellite galaxy. The colour indicates the different samples.

from Eq. 1 we calculate the relative mass uncertainty as:

$\frac{\sigma(M)}{M}=\frac{\sigma\left(v_{\mathrm{tot}}^{2} r_{\mathrm{GC}}^{0.02}\right)}{\left\langle v_{\mathrm{tot}}^{2} r_{\mathrm{GC}}^{0.02}\right\rangle}$

where $\sigma$ is the standard deviation obtained using 2000 Monte Carlo draws, for each matched subhalo in a given ELVIS simulation. These values can be under- or over- estimated depending on the velocity that the subhalo happened to have in that given simulation. We then calculate the average $\sigma(M) / M$ for each subhalo over the 24 simulations to remove this effect, such that we are left with the effect imprinted by the observational uncertainties. The results are shown in Fig. 8: as expected the uncertainties are larger for the most distant galaxies, albeit with some scatter.

The galaxies causing the largest $\sigma(\mathrm{M}) / \mathrm{M}$ are Leo IV, Leo V, Pisces II, Hydra II, Columba I, Aquarius II, Reticulum III, Canes Venatici II. These correspond to a $\sigma(M) / M \gtrsim$ 0.75. Besides these, Horologium II suffers from a large difference between the preferred proper motions derived by Fritz et al. (2019) and Pace \& Li (2019). Excluding from the mass determination the sub-haloes that would be associated to these galaxies leads to the results shown in the lower panel of Fig. 7. The average correction factor is now smaller ${ }^{9}$, typically 1.4 , and the average scatter over the different simulations is now only 0.07 . That implies that the relative overall uncertainty is only 0.005 larger than without added uncertainties. An even stricter exclusion of galaxies is not advisable as it would lead to the exclusion of the most distant galaxies, which are especially useful to measure the mass within large radii.

We also tested less strict cuts, but found that the obtained masses overlap well with the one obtained with our chosen cut.

\subsection{Conclusions about biases}

We conclude that the biases in the mass estimators are likely due to deviations of the simulations from the assumptions

9 The correction factor depends on mass, being usually smaller for smaller masses. 
of the mass estimator. The gravitational potential does not appear to be the culprit, as it is well approximated by a single power-law in the radial range considered. On the other hand, the radial density of the tracers, the subhalos, does not follow well a single power-law, as discussed in Section 3.3. For example, the simulations present a central deficit of subhaloes with respect to the expectations from a single powerlaw fitted to the sub-haloes radial number density distribution. A reason for this deficit is disruption by tidal forces due low resolution in the simulations (van den Bosch et al. 2018; Errani \& Peñarrubia 2019). If that is the case, it is likely that the subhalos found at small distances are those close to their pericentre, and thus are biased towards larger velocities than assumed in the scale free Wa10 mass estimator. The behaviour of the sub-haloes velocity anisotropy, which is slightly tangential in the inner regions and radial further out (with a difference of $-0.18 \pm 0.04$ between the two), is likely due to this effect.

Since we are using the simulations to tackle the bias in the MW mass determination when applying the Wa10 estimator, and deduce a correction factor, it is important that simulations closely resemble the real systems. On one hand, real satellite galaxies undergo baryonic effects that might enhance tidal disruption (see e.g. D'Onghia et al. (2010), Kelley et al. (2019), Samuel et al. (2019)), and that, by definition, are not included in dark-matter only simulations. On the other hand, a known issue with simulations is that subhalos are destroyed too rapidly, see van den Bosch et al. (2018); Errani \& Peñarrubia (2019). Thus these two effects act in opposite directions. Observationally, the radial trend in $\beta$ hints to a more efficient destruction than in DM only simulations, while the high (concentrated) value of $\gamma$ hints to the opposite.

We conclude that there is no clear evidence that we under- or overestimate the correction factor and thus we use the values calculated in Section 4.2. It is possible that the estimate of the correction factor is sub-optimal, due to mismatches between the simulations and the true Milky Way: in this regard, it would be useful to revise this determination when hydrodynamic simulations will be able to provide a satisfactory match to the population of Milky Way satellite galaxies.

\section{ASSUMPTIONS REGARDING SATELLITES OF THE LMC}

In this section we lay the ground for testing the effect that the LMC has on the MW mass estimate, in two respects: accounting for the fact that some of the galaxies in the sample might be former LMC satellites; and taking into consideration the gravitational pull that a massive LMC is expected to exert on the MW (Gómez et al. 2015).

As a first step, we determine how the mass changes when excluding galaxies that are likely to be former satellites of the LMC. These systems share similar orbits as their former host, and thus their inclusion would lead to an underestimated uncertainty if they are assumed to be independent tracers. Since we use cosmological simulations to estimate the uncertainty on the MW mass estimate, the effect of group infall is in principle included; therefore, we do not exclude small (and uncertain) groups such as the potential
Crater-Leo II group from our sample (e.g. Fritz et al. 2018). The LMC is, however, an unusually large satellite (satellites as massive as the LMC are rare in observations and simulations, e.g. Liu et al. 2011, Boylan-Kolchin et al. 2010). We therefore exclude its satellites in this test, but we do include the LMC itself, since one tracer should be used for a group.

The membership in the LMC associated group is still uncertain for galaxies with large proper motion uncertainties, see Fritz et al. (2019), which also implies that these systems are among those that are not included in our final sample. On the other hand, even when a proper motion is well known, other factors such as the mass of the LMC itself do play a role (Kallivayalil et al. 2018).

We classify the SMC, Hyi I, Car II, Car III and Horo I as "probable" LMC group members (Kallivayalil et al. 2018), and Dra II, Hydra II (Kallivayalil et al. 2018), Phoenix II as "possible" members (Fritz et al. 2019). The possible members do not agree with the properties of the expected debris in Kallivayalil et al. (2018), but this may be due to differences between the LMC-analogue used in that work and the real LMC. There are more potential members in the view of Pardy et al. (2019), such as Fornax and Carina I, but since these galaxies are now close to apocentre (while the LMC is at pericentre) we think that an association is unlikely (see also Sales et al. 2011). While they share the orbital plane of the $\mathrm{LMC}^{10}$, they do not have the other properties that would classify them as former satellites.

We end with three different samples: 1 . all satellites, 2 . a sample that excludes probable former LMC satellites and 3. a sample that excludes probable and possible former LMC satellites ("excludes all former LMC satellites").

To include the effects of the LMC gravitational pull on the MW, it would be ideal to have many analogues of $\mathrm{LMC}+\mathrm{MW}$ in simulations, which is not yet possible. As an approximation we calculate the distance and total velocities of the satellite galaxies compared to the barycentre of MW and LMC. We tried several options for the relative LMC mass, but to give an idea of the potential size of the effect we use the most extreme plausible option, i.e. that the LMC has a fourth of the mass of the MW. That is slightly larger than recent estimates but not ruled out: Peñarrubia et al. (2016) obtained a mass ratio of $0.19 \pm 0.05$ from mostly LOS motions and timing arguments; the number of former LMC satellites points to a ratio of $0.18 \pm 0.09$ (Fritz et al. 2019); and the Orphan stream points to a mass ratio of between 0.12 to 0.19 (Erkal et al. 2019).

Galaxies close to the Milky Way can adapt to the changing velocity of the MW, with the transition being at about $30 \mathrm{kpc}$ (Erkal et al. 2019). Thus, galaxies further in should follow the Milky Way, even when considering the barycenter of the LMC+MW system. Since the transition is not sharp, we define a parameter for the strength of the reflex motion $\left(c_{\text {refl }}\right)$ calculated as:

$$
c_{\text {refl }}=\left(\tanh \left(r_{\mathrm{GC}}[\mathrm{kpc}]-30\right) / 10+1\right) / 2
$$

When $c_{\text {refl }}=0$ the velocity and position of a satellite is relative to the Milky Way, when it is 1 the velocity and position of a galaxy is relative to the barycentre of the LMC $+\mathrm{MW}$

10 They are members of the vast polar structure (Pawlowski et al. 2012). 
system. It is larger than 0.5 for most galaxies. For the closest galaxy Sagr I, it is 0.25 . Therefore, the difference compared to using barycentric for all is small.

Since this correction is only approximate, we provide the MW mass estimate obtained in this way in addition to the case of referring positions and velocities of the satellite galaxies only to the MW.

We combine these two cases with the above outlined three cases regarding former LMC satellites. Thus, we have in total six different combinations, see Table 1.

\section{THE MASS OF THE MILKY WAY}

In this section we obtain the mass of the MW and compare with previous results, as well as comparing the mass we obtain as a function of radius with simulations.

\subsection{Derivation of the Milky Way mass}

We now apply the Wa10 mass estimator to obtain the mass of the Milky Way, by using Eq. 1 . We adjust $M_{<=\text {out }}$ by the correction factor $\left(f_{\text {cor }}\right)$ to the correct mass $M_{<=\text {out cor }}$ :

$M_{<=\text {out cor }}=\frac{M_{<=\text {out }}}{f_{\text {cor }}}=\frac{1}{G f_{\text {cor }}} \frac{\alpha+\gamma-2 \beta}{3-2 \beta} r_{\mathrm{GC} \mathrm{out}}^{1-\alpha}\left\langle v_{\mathrm{tot}}^{2} r_{\mathrm{GC}}^{\alpha}\right\rangle$.

We use the distributions for $\beta$ and $\gamma$ obtained in Sections 3.2 and 3.3. The mass is then obtained in an iterative process, since both the correction factor and the parameter $\alpha$ depend on the mass itself. First we iterate until $\alpha$ converges, then add the mass independent uncertainty on $\alpha$ of 0.0706 (see Section 3.1) as a Gaussian. The number of iterations is set to be high enough to ensure that the mass changes by less than $0.5 \%$ at the end of the final iteration. The obtained mass is then scaled with the correction factor obtained in Section 4.2 to get the mass at the outermost radius. After five iterations we have a distribution of mass values that depend on $\alpha, \beta$ and $\gamma$. We then add the uncertainty of the correction factor obtained in Section 4.2 as a Gaussian uncertainty. We repeat this process for the different samples, i.e. those with and without LMC associated satellites, as outlined in Section 5.

The values obtained for the MW mass for the different combination of cases are summarised in Table 1. In all cases we calculated masses from the inner (19 to $64 \mathrm{kpc}$ ), outer (77 to $273 \mathrm{kpc}$ ), and full (19 to $273 \mathrm{kpc}$ ) radial ranges, showing the mass within the distance of the outermost satellite. Fig. 9 shows the distribution of masses for the most extreme cases: the mass derived from the full sample, centred on the MW (left panel); and from the most reduced sample, relative to the centre of mass of the MW-LMC system, and disregarding all former LMC satellites (right panel).

When all the tracer galaxies are used, we obtain the largest value of the mass within $273 \mathrm{kpc}$ (full radial range), $M_{273}=1.84_{-0.36}^{+0.40} \times 10^{12} \mathrm{M}_{\odot}$, while by excluding all potential LMC satellites, and computing the positions and velocities relatively to the centre of mass of the MW-LMC system, the obtained mass is the smallest possible with $M_{273}=1.28_{-0.27}^{+0.30} \times 10^{12} \mathrm{M}_{\odot} \cdot{ }^{11}$ While it is unlikely that in-

11 We note that here as in the rest of the paper $\mathrm{x}$ stands for the full mass within X (e.g. 273) kpc. cluding all the galaxies centred on the MW is correct, since it is known that the LMC is on an eccentric orbit which biases the estimates towards large values, it is difficult to assess which of the other five cases is preferable: we therefore average them (see Table 1) and add to the uncertainty the scatter over the median masses. The result is $M_{273}=1.43_{-0.32}^{+0.35} \times 10^{12} \mathrm{M}_{\odot}$, which is our preferred value for the MW mass within $273 \mathrm{kpc}$.

The mass derived within the outer radial range depends less on the choice of the sample, since there are less former LMC satellites in the outskirts of the MW: the outer mass varies only between 1.37 and $1.53 \times 10^{12} \mathrm{M}_{\odot}$, with an average of $M_{273}=1.45_{-0.35}^{+0.37} \times 10^{12} \mathrm{M}_{\odot}$. The two different estimates of the MW mass at $273 \mathrm{kpc}$ agree well, although the correction factor is different and the sample differs by half. Within 64 $\mathrm{kpc}$, instead, the mass varies from 0.51 to $0.73 \times 10^{12} \mathrm{M}_{\odot}$, with an average of $M_{64}=0.58_{-0.14}^{+0.15} \times 10^{12} \mathrm{M}_{\odot}$.

Finally, we convert the mass within $273 \mathrm{kpc}$ to the virial mass. Since our sample nearly extends to the virial radius, a large extrapolation is not required. We tried several methods. Firstly by using the virial mass and mass profile given by ELVIS simulations. We also used a typical NFW profile which concentration-mass relation from Planck cosmology and overdensity of 97 . We then used an NFW profile with a value of concentration which is twice the dark matter only value, since the contraction in Milky Way-like hydrodynamic simulations is found to be higher than in dark matter only simulations at this mass (see Fig. 4 in Di Cintio et al. 2014).

The difference between the largest and smaller estimates of the virial mass is $6 \%^{12}$, significantly less than our overall uncertainty. We use the median value, which is obtained with the NFW extrapolation and twice the standard concentration. This results in $M_{\mathrm{vir}}=1.51_{-0.40}^{+0.45} \times 10^{12} M_{\odot}$, which is our preferred value for the virial mass of the Milky Way, corresponding to a virial radius of $R_{\mathrm{vir}}=308 \pm 29 \mathrm{kpc}$.

We then explore which of the parameters of the mass estimator most affects the uncertainty on the mass. We vary the different power-law indexes separately for the full sample, setting the fixed indices to their median values. We obtain the following relative uncertainties: for $\alpha 8.1 \%$; for $\beta 4.3 \%$; for $\gamma 4.4 \%$. Thus, uncertainty in $\alpha$, the galaxy mass profile, is what matters the most, while the uncertainty in the tracer profile $(\gamma)$ and the anisotropy parameter $(\beta)$ are of similar, lower, importance. Therefore, although the $\beta$ uncertainty is large, its impact is relatively minor because we use full velocities and not only radial (approximately line-of-sight) or tangential (proper motions) velocities. This is in agreement with the analytic derivation, shown in Di Cintio et al. (2012), of the relative uncertainties of each parameter and their impact on the mass estimators uncertainty.

The uncertainties on these three free parameters all together contribute an uncertainty of $13.5 \%$, which is more than expected from simply adding the uncertainties individually, since such parameters are correlated: a change in $\beta$ and $\gamma$ modifies the expected mass which in turn changes $\alpha$, that depends on the mass. Thus, their combined contri-

12 Our definition of the virial mass assumes $\Delta=97$. Other 'virial' masses are indicated when used in comparisons, for example $M_{\mathrm{vir}, 200}$. The virial masses include the full mass, not only DM. 
Table 1. Mass estimates for the various cases. Col. 2 lists the selection of galaxies, i.e. whether former LMC satellites are excluded; col. 3 the reference frame, either MW only or the barycentre of MW and LMC; col. 4 gives the number of galaxies in the full, inner and outer sample and col. 5, 6, 7 give the median and the range of the $68.3 \%$ confidence interval within $\mathrm{X}$ kpc derived from the full sample, inner sample and outer sample.

\begin{tabular}{|c|c|c|c|c|c|c|}
\hline case & galaxy selection & reference frame & number of galaxies & $M_{273}\left[M_{\odot}\right]$ & $M_{64}\left[M_{\odot}\right]$ & $M_{\text {outer }, 273}\left[M_{\odot}\right]$ \\
\hline 1 & full sample & MW & $36 / 20 / 16$ & $1.84_{-0.36}^{+0.40} \times 10^{12}$ & $0.73_{-0.15}^{+0.16} \times 10^{12}$ & $1.53_{-0.33}^{+0.35} \times 10^{12}$ \\
\hline 2 & full sample & barycentric & $36 / 20 / 16$ & $1.53^{+0.34} \times 10^{12}$ & $0.63^{+0.15} \times 10^{12}$ & $1.46^{+0.33} \times 10^{12}$ \\
\hline 3 & without probable former LMC satellites & MW & $31 / 16 / 15$ & $1.54_{-0.33}^{+0.31} \times 10^{12}$ & $0.62_{-0.14}^{+0.15} \times 10^{12}$ & $1.49_{-0.34}^{+0.36} \times 10^{12}$ \\
\hline 4 & without probable former LMC satellites & barycentric & $31 / 16 / 15$ & $1.36_{-0.3}^{+0.33} \times 10^{12}$ & $0.54_{-0.12}^{+0.13} \times 10^{12}$ & $1.48_{-0.34}^{+0.36} \times 10^{12}$ \\
\hline 5 & without all former LMCsatellites & MW & $29 / 15 / 14$ & $1.43_{-0.31}^{+0.34} \times 10^{12}$ & $0.6_{-0.14}^{+0.15} \times 10^{12}$ & $1.37^{+0.35} \times 10^{12}$ \\
\hline 6 & without all former LMCsatellites & barycentric & $29 / 15 / 14$ & $1.28_{-0.27}^{+0.31} \times 10^{12}$ & $0.51_{-0.11}^{+0.12} \times 10^{12}$ & $1.45_{-0.35}^{+0.38} \times 10^{12}$ \\
\hline
\end{tabular}
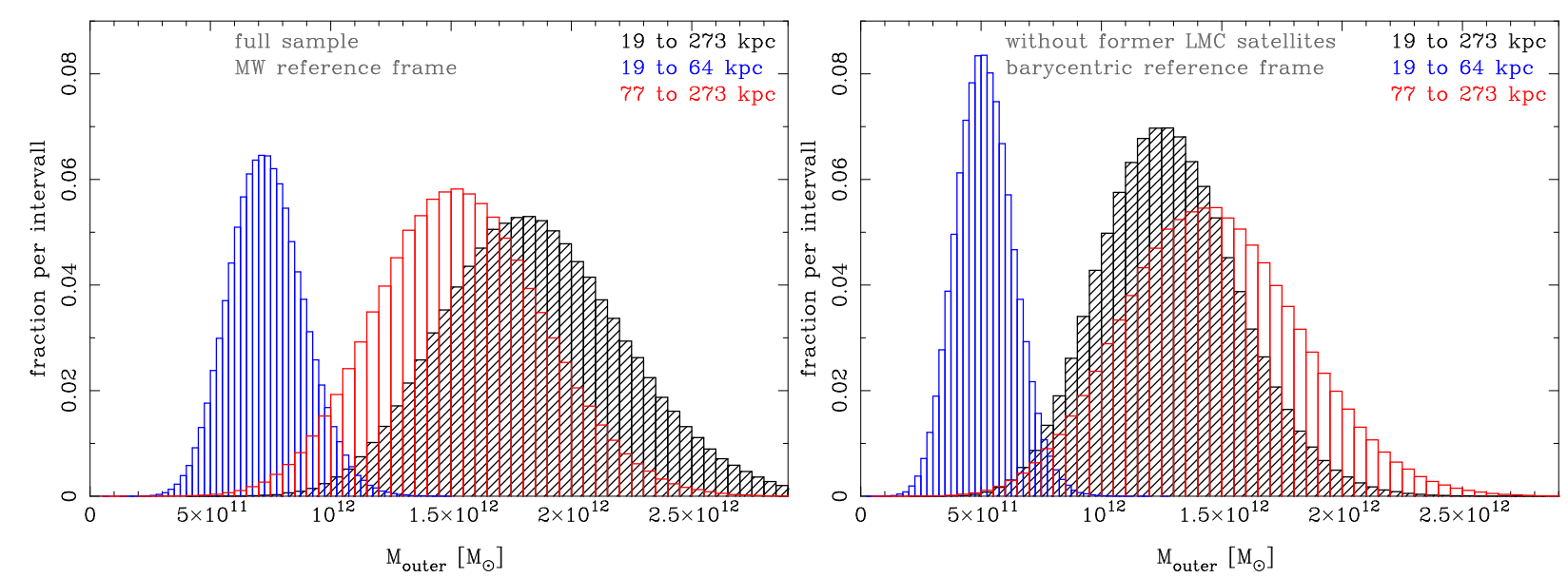

Figure 9. Probability histograms of the Milky Way mass calculated within the inner, outer, and full radial ranges, shown in blue, red and black, respectively. In the left panel we show results for our full sample of satellite galaxies, for which we obtained a value of the MW mass within $273 \mathrm{kpc}$ of $M_{273}=1.84_{-0.36}^{+0.40} \times 10^{12} \mathrm{M}_{\odot}$, while in the right panel we show results for the most restrictive sample in which all possible former satellites of the LMC are excluded, and for which the derived MW mass reads $M_{273}=1.28_{-0.27}^{+0.30} \times 10^{12} \mathrm{M}_{\odot}$. In this last case, the velocities and distances of the tracers are relative to the centre of mass of the MW-LMC system. We show the two extreme estimates of mass: other cases provide values of mass in between these two.

bution is nearly the same as the uncertainty caused by the calibration of the mass estimator itself, which is $15 \%$. Improved determinations of $\alpha, \beta$ and $\gamma$ would therefore result in significant improvement in the application of the Wa10 mass estimator.

\subsection{Comparison with simulations}

We now compare our estimates of mass at different radii with the MW mass profile coming from cosmological simulations.

From the ELVIS suite we select DM-only halos whose mass at $273 \mathrm{kpc}$ is within $1-\sigma$ interval of our mass estimate, as shown in Fig. 10: the virial mass of such halos range between 1.06 and $1.90 \times 10^{12} \mathrm{M}_{\odot}$, and they follow an NFW profile.

At $64 \mathrm{kpc}$, instead, our measurement of the MW mass lies above the average of the DM-only predictions: that is expected because DM-only halos neglect the contribution of baryons. This results in an even clearer disagreement at $19 \mathrm{kpc}$ and further in, where the contribution of baryons is increasingly important.

We therefore compare to hydrodynamical simulations, for which we use the NIHAO and MaGICC simulations, considering only those simulated MWs within $1 \sigma$ of the MW' observed $v_{\text {circ }}$ value at $19 \mathrm{kpc}$. This gives us five simulations, that span a virial mass range between 1.06 and $1.40 \times 10^{12}$
$\mathrm{M}_{\odot}$. We see that further out than $19 \mathrm{kpc}$ most of these hydro simulations are below our most likely estimate, however 4 out of 5 still lie within our uncertainty at $273 \mathrm{kpc}$.

Over-all, the mass profile derived from our measurements agrees well with recent hydro-dynamical simulations of the MW, despite of the fact that we did not use for our mass estimates. This shows that the simplifications present in the simulations and the assumptions made for our mass estimates probably do not have a large impact on the mass estimate.

\subsection{Comparison with previous results}

Fig. 10 shows the comparison of our measurement of the MW mass with those from the literature. We concentrate on those measurements over a similar radial range as our work $(\mathrm{r}>30 \mathrm{kpc})$, that do not use large extrapolations and/or that do not combine different types of tracers that might follow a different radial distribution like Battaglia et al. (2005), Dehnen et al. (2006), Karukes et al. (2019) and Cautun et al. (2019). We list the measurements sorted by the tracer used, in order to explore whether the choice of tracer matters for the derivation of the mass.

We first note that our determination agrees well with the virial mass of $M_{\mathrm{vir}}=1.3 \pm 0.3 \times 10^{12} \mathrm{M}_{\odot}$, shown as a red square in Fig. 10, as estimated in the review of 


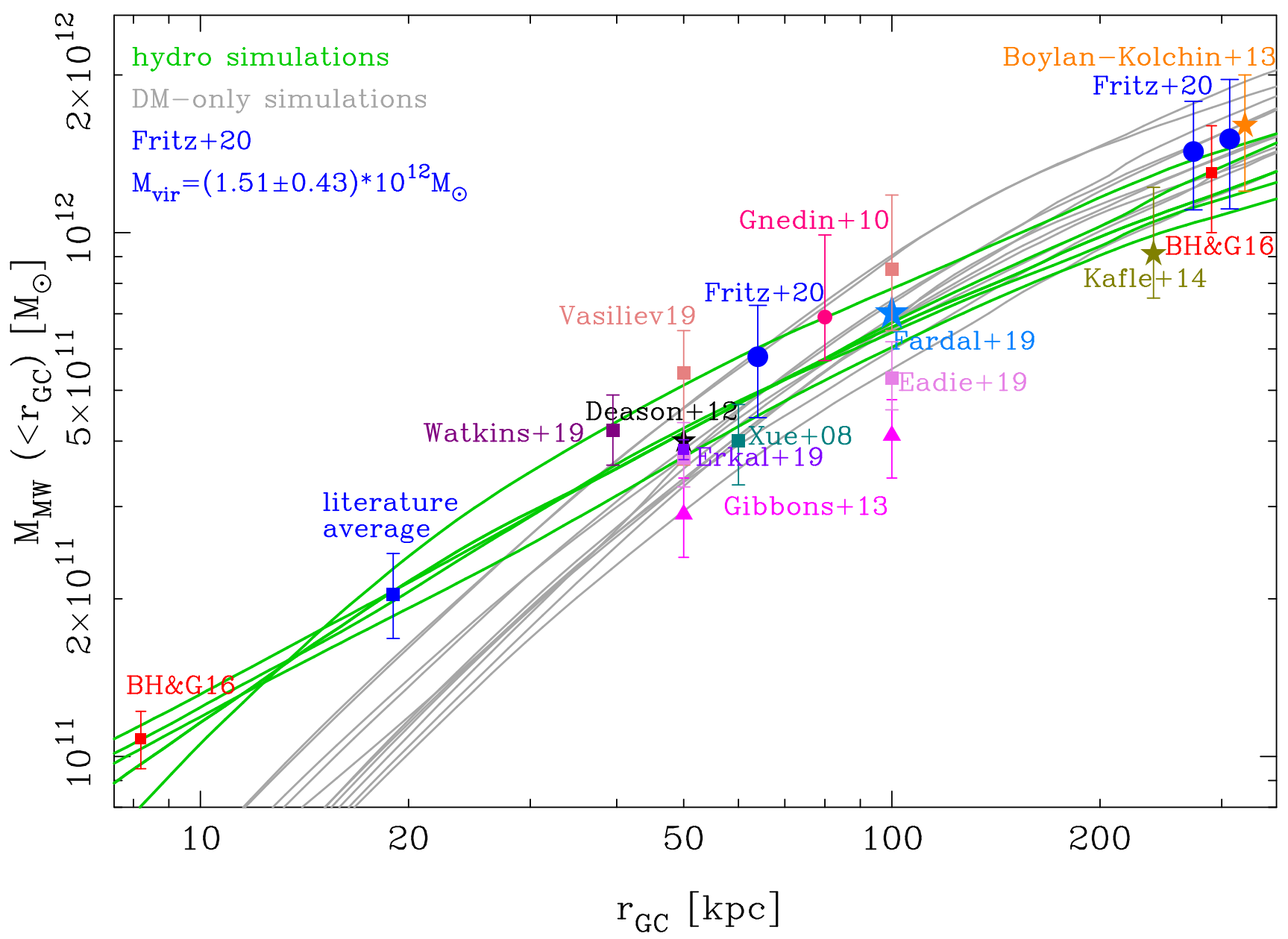

Figure 10. Radial distribution of the mass of the Milky Way, in Galactocentric coordinates. We show with blue dots our measurements at 64,273 and $293 \mathrm{kpc}$, where the outer most point is the virial radius. $1 \sigma$ uncertainties are also shown. The blue box at $19 \mathrm{kpc}$ is our average estimate from Küpper et al. (2015), Bovy et al. (2016), Watkins et al. (2019). With grey lines we show the mass profiles from the DM only ELVIS simulations, which match within $1 \sigma$ our mass at $273 \mathrm{kpc}$. With green lines we show the total mass from NIHAO and MaGICC hydrodynamical simulations, which match within $1 \sigma$ the mass profile at $19 \mathrm{kpc}$. The remaining points are previous literature results (Vasiliev 2019; Eadie \& Jurić 2019; Watkins et al. 2019; Kafle et al. 2014; Deason et al. 2012; Fardal et al. 2019; Xue et al. 2008; Gibbons et al. 2014; Bland-Hawthorn \& Gerhard 2016; Boylan-Kolchin et al. 2013), based on different methodologies (see text for more detail). The estimates of Boylan-Kolchin et al. (2013) and Kafle et al. (2014) are two of the most extreme virial mass estimates, see text for more values in between.

Bland-Hawthorn \& Gerhard (2016), using an average of halo stellar kinematic measurements (see also Wang et al. 2015a and Wang et al. 2019 for other reviews).

The advantage of using halo stars for this type of determinations is that they are more numerous than other tracers, such as globular clusters, satellite galaxies or streams. Xue et al. (2008) obtain from the SDSS BHB stars a mass at $60 \mathrm{kpc}$ of $M_{60}=0.4 \pm 0.07 \times 10^{12} \mathrm{M}_{\odot}$. Deason et al. $(2012)$ obtain from SDSS BHB stars a value of $M_{50}=0.4 \times 10^{12} \mathrm{M}_{\odot}$. Gnedin et al. (2010) uses also BHB stars from a dedicated survey to measure $M_{80}=0.69_{-0.12}^{+0.30} \times 10^{12} \mathrm{M}_{\odot}$. Kafle et al. (2014) use K-giant stars, in addition to BHB ones, out to $155 \mathrm{kpc}$, to obtain $M_{\mathrm{vir}}=0.91_{-0.16}^{+0.31} \times 10^{12} \mathrm{M}_{\odot}$, corresponding to a $R_{\text {vir }}$ of $\sim 239 \mathrm{kpc}$, shown as a green star in Fig. 10 . We note that this value is based on $R_{0}=8.5 \mathrm{kpc}$, and that reducing $R_{0}$, as suggested by Bland-Hawthorn \& Gerhard (2016) and Gravity Collaboration et al. (2019), would increase the value of the mass. Huang et al. (2016) obtain
$M_{\text {vir }}=0.97_{-0.08}^{+0.07} \times 10^{12} \mathrm{M}_{\odot}$, mainly from line-of-sight velocities of giant stars out to distances of $100 \mathrm{kpc}$,

The escape speed of local stars can also constrain the virial mass. Using this method, Piffl et al. (2014) obtain $M_{\mathrm{vir}, 200}=1.6_{-0.4}^{+0.5} \times 10^{12} \mathrm{M}_{\odot}$ using the RAVE survey. Monari et al. (2018) use a similar method based on counter rotating stars from Gaia DR2 to obtain $M_{\mathrm{vir}, 200}=1.28_{-0.5}^{+0.68} \times$ $10^{12} \mathrm{M}_{\odot}$. Similarly, Hattori et al. (2018) use high velocity stars from Gaia DR2 to obtain $M_{\mathrm{vir}, 200} \approx 1.4 \times 10^{12} \mathrm{M}_{\odot}$. Deason et al. (2019) also use high velocity stars from Gaia DR2 to obtain $M_{\text {vir,200 }}=1.00_{-0.24}^{+0.31} \times 10^{12} \mathrm{M}_{\odot}$. When better accounting for biases, especially those affecting the halos that are dominated by early mergers, by using simulations from Grand et al. (2019), Deason et al. (2019) get an increased value of $M_{\text {vir,200 }}=1.29_{-0.47}^{+0.37} \times \mathrm{M}_{\odot}$. We note that since all these five virial masses are at an overdensity of 200 , so need to be multiplied by about 1.19 to compare the other virial masses cited, see Bland-Hawthorn \& Gerhard (2016). With this correction, their range covers our value. 
Stellar streams are powerful probes of the MW gravitational potential (Johnston et al. 1999). Mainly from the precession of the Sagittarius stream, Gibbons et al. (2014) derive low masses for the $\mathrm{MW}$ at 50 and $100 \mathrm{kpc}, M_{50}=0.29 \pm$ $0.05 \times 10^{12} \mathrm{M}_{\odot}$ and $M_{100}=0.41 \pm 0.07 \times 10^{12} \mathrm{M}_{\odot}$. This method is not free of assumptions, especially regarding the form of the underlying potential: Fardal et al. (2019) use similar properties of the Sagr stream but different potential slopes and shapes for the halo of the MW to obtain a preferred mass $M_{100}=0.70 \times 10^{12} \mathrm{M}_{\odot}$. Erkal et al. (2019) use the Orphan stream to obtain a mass of $M_{50}=0.39 \pm 0.02 \times 10^{12} \mathrm{M}_{\odot}$. Their model includes the influence of the LMC, although the shape of the LMC potential due to the infall onto the MW is not taken into account; furthermore, the dark matter wake introduced by the gravitational influence of the LMC might cause deviations of the potential of the MW from a prolate/oblate NFW profile (Garavito-Camargo et al. 2019).

Globular clusters are rather abundant in the inner halo and thus a useful tracer of those regions. Watkins et al. (2019) used halo globular clusters with proper motions from Gaia DR2 (Gaia Collaboration et al. 2018a,b) and HST (Sohn et al. 2018), to obtain a value of $M_{39.5}=0.42_{-0.06}^{+0.07} \times$ $10^{12} \mathrm{M}_{\odot}$ using their Wa10 mass estimator. Vasiliev (2019) use Gaia DR2 to measure the proper motion of globular clusters out to $\sim 100 \mathrm{kpc}$, and they then use distribution functions to obtain $M_{50}=0.54_{-0.07}^{+0.11} \times 10^{12} \mathrm{M}_{\odot}$ and $M_{100}=0.85_{-0.20}^{+0.33} \times 10^{12} \mathrm{M}_{\odot}$. Eadie \& Jurić (2019) use the data sets of Sohn et al. (2018), Gaia Collaboration et al. (2018b), Vasiliev (2019) outside of $15 \mathrm{kpc}$ and the method of Eadie et al. (2015) to obtain $M_{50}=0.37_{-0.04}^{+0.06} \times 10^{12} \mathrm{M}_{\odot}$ and $M_{100}=0.53_{-0.07}^{+0.09} \times 10^{12} \mathrm{M}_{\odot}$. The difference between the last works show that even when the same proper motions are used, other assumptions, such as the radial distribution of the tracer population, can change the obtained value of the MW mass.

In the past the mass obtained using dwarf galaxies often depended on whether or not Leo I was assumed to be a bound satellite, see e.g. Kulessa \& Lynden-Bell (1992). When assumed to be bound, its HST proper motion leads to a mass of $M_{\mathrm{vir}}=1.6 \pm 0.4 \times 10^{12} \mathrm{M}_{\odot}$ (Boylan-Kolchin et al. 2013). That is slightly higher than our estimate but consistent at the $1 \sigma$ level.

Watkins et al. (2010) obtain a preferred estimate of $M_{300}=2.7 \times 10^{12} \mathrm{M}_{\odot}$, using the Wa10 mass estimator on a sample of 6 dwarf galaxies with and 20 without proper motions as was available in the data at that time. They found a $\beta=-4.5$. The recently improved determination of $\beta$, which is still tangential but less than the value reported in Watkins et al. (2010), leads to a lower mass estimate. Our analysis also suggests that the Wa10 value was also relatively high due to the large value of $\gamma$ coming from an incomplete sample of dwarfs, and by the assumption that the mass estimator is unbiased when applied to samples in which galaxies close to their pericenters are overrepresented.

Using HST proper motions of classical dwarfs and the LMC along with the Illustris simulation to match specific angular momenta of the observed dwarfs and their analogues in the simulation, Patel et al. (2018) obtain $M_{\mathrm{vir}}=0.96_{-0.28}^{+0.29} \times$ $10^{12} \mathrm{M}_{\odot}$. Matching classical dwarf galaxy distribution functions, constructed using HST and Gaia proper motions, and the EAGLE simulations using a distribution function by fit- ting angular momenta and specific energy, Callingham et al. (2019) obtained $M_{\text {vir,200 }}=1.17_{-0.15}^{+0.21} \times 10^{12} \mathrm{M}_{\odot}$. Upscaled to $M_{\text {vir }}$ results in $\approx 1.39 \times 10^{12} \mathrm{M}_{\odot}$. Very recently Li et al. (2019) used a similar sample of dwarf galaxies and a distribution function method based on the the EAGLE simulation to obtain $M_{\mathrm{vir}, 200}=1.23_{-0.18}^{+0.21} \times 10^{12} \mathrm{M}_{\odot}$. Upscaled to $M_{\mathrm{vir}}$ this converts in $\approx 1.46 \times 10^{12} \mathrm{M}_{\odot}$, well matching our result.

In summary, there is no clear trend of low or high masses depending on the particular tracer, with each being used to recover a range of masses, although it can be noted that the masses obtained from streams do not cover the high mass tail of the various estimates. Our estimated mass of $M_{\mathrm{vir}}=1.51_{-0.40}^{+0.45} \times 10^{12} M_{\odot}$ is intermediate between literature estimates (where the largest one is from the work by Boylan-Kolchin et al. 2013 and the smallest one by Kafle et al. 2014).

The way that the LMC is accounted for (or not accounted for) could explain at least part of the differences in the various mass estimates. It is likely that our MW mass estimate includes the mass of the LMC at least partly, since the majority of our tracers are at larger distances than the LMC and thus for them the force of the LMC acts in about the same direction as the force of the MW. The LMC alone is more massive (about 20\% of the MW mass) than a median subhalo population in ELVIS, which amount to just $10 \%$ of the main halo. Thus, the LMC is more important than the typical subhalos. The LMC might also explain why our mass at $64 \mathrm{kpc}, 0.58_{-0.14}^{+0.15} \times 10^{12} \mathrm{M}_{\odot}$, is larger than the mass by Erkal et al. (2019) who included the LMC explicitly. If our virial mass includes the LMC our results would then fit better to the timing result of Peñarrubia et al. (2016), who obtain $M_{\mathrm{MW}}=1.04_{-0.23}^{+0.26} \times 10^{12} \mathrm{M}_{\odot}$ and $M_{\mathrm{LMC}}=$ $0.25_{-0.08}^{+0.09} \times 10^{12} \mathrm{M}_{\odot}$.

\section{CONCLUSIONS AND SUMMARY}

The mass of the Milky Way is still surprisingly uncertain, notwithstanding the impressive amount of literature on the topic and the variety of approaches taken. Due to their large distances, dwarf galaxies are a promising tracer to sample the mass of the MW to its outskirts. Thanks to Gaia DR2, there are now systemic proper motions and line-of-sight velocities available for 45 galaxies within $280 \mathrm{kpc}$ (Fritz et al. 2018, 2019; Gaia Collaboration et al. 2018b; Carlin \& Sand 2018; Torrealba et al. 2019; Longeard et al. 2019); this leverages the mass determination by also providing information on the velocity anisotropy of the tracer population.

We use this Gaia DR2 data set to estimate the mass of the Milky Way using the scale free mass estimator of Watkins et al. (2010). We determine the potential index $\alpha$, the anisotropy parameter $\beta$, and the tracer density index $\gamma$ parameter for the Milky Way. We determine, from a likely complete sample (15 galaxies with $\left.\mathrm{M}_{V}<-5.9\right)$, that $\gamma=2.11 \pm 0.23$, but we note that the galaxy number density profile is not well fit by a single power-law, with a deficit of satellites within $18 \mathrm{kpc}$. We obtain $\beta=-0.67_{-0.62}^{+0.45}$, or $\beta=-0.21_{-0.51}^{+0.37}$ when we exclude from the sample all possible former satellites of the LMC. We determine $\alpha$ iteratively, combining a direct calculation of observed mass within 19 $\mathrm{kpc}$ from circular velocity determinations and estimates of 
the mass within $273 \mathrm{kpc}$ from the Wa10 estimator. This implies that the value of $\alpha$ depends on the MW mass. For our derived Milky Way mass $\left(M_{273}\right)$ and its uncertainty, it follows that $\alpha=0.27_{-0.11}^{+0.12}$.

We use cosmological simulations to check for biases introduced by observational uncertainties and by the radial distribution of tracers. We find that the mass obtained is biased even without observational uncertainties. This appears to be the result of the distribution of subhalos not being scale-free, as assumed in the Wa10 estimator, but having a deficit of satellites in the inner region where galaxies are more likely close to their pericenters. We correct for that bias by applying a correction factor, which is included in the following listed results.

\section{Summarising our main results:}

- Observational uncertainties lead to an increase in the value of the recovered mass with, as expected, larger errors. We devise a method to identify which galaxies introduce the largest uncertainties, due to their proper motion errors, and exclude them, resulting in a sample of 36 galaxies.

- We use different samples to assess the importance of former satellites of the LMC and reference frames to account for the reflex motion of the MW due to the LMC.

- Using the full radial range of galaxies, we obtain a mass of $1.43_{-0.32}^{+0.35} \times 10^{12} \mathrm{M}_{\odot}$ within $273 \mathrm{kpc}$. This is consistent with the value determined when using only the outer half of the sample.

- From the inner half of the sample we obtain $0.58_{-0.14}^{+0.15} \times$ $10^{12} \mathrm{M}_{\odot}$ within $64 \mathrm{kpc}$.

- We derive a virial mass of $M_{\text {vir }}=1.51_{-0.40}^{+0.45} \times 10^{12} M_{\odot}$ within $\mathrm{R}_{\mathrm{vir}}=308 \pm 29 \mathrm{kpc}$ for an overdensity of 97 , by averaging the various cases (i.e. with or without former LMC satellites).

- We find that, of the scaling parameters, the mass profile $\alpha$ has the biggest impact on the mass. When combined, the three scaling parameters have nearly the same importance as the mass estimator calibration and the intrinsic uncertainty.

The mass determination from this work is slightly larger than the average of literature values given by Bland-Hawthorn \& Gerhard (2016), and thus provides support for an intermediate mass for the Milky Way (Fritz et al. 2018; Watkins et al. 2019), disfavouring a low mass Milky Way solution to the 'too big to fail' (TBTF) problem (Boylan-Kolchin et al. 2011; di Cintio et al. 2011; Wang et al. 2012; Vera-Ciro et al. 2013), indicating the need for other solutions such as feedback (e.g. Brook \& Di Cintio 2015; Wetzel et al. 2016) or modifications to CDM (e.g. Hui et al. 2017).

Adopting a Milky Way stellar mass of $6 \times 10^{10} \mathrm{M}_{\odot}$ from Bland-Hawthorn \& Gerhard (2016), we can compare to abundance matching results: our inferred virial mass places the Milky Way at the expected position in the $\mathbf{M}^{*}-\mathbf{M}_{\text {halo }}$ relation of Kravtsov et al. (2018), while, interestingly, all other abundance matching studies would predict a larger value for the Milky Way mass (see Behroozi et al. 2019; Somerville et al. 2018; Wechsler \& Tinker 2018; Rodríguez-Puebla et al. 2017 for review).

Our result can also be compared with the mass of M31, for which derived masses range from about $M_{\mathrm{vir}}=0.8 \pm 0.1 \times$
$10^{12} \mathrm{M}_{\odot}$ (Kafle et al. 2018), over $M_{\mathrm{M} 31300}=1.40 \pm 0.43 \times$ $10^{12} \mathrm{M}_{\odot}$ (Watkins et al. 2010 ) to $M_{\text {vir }}=2.1 \pm 0.5 \times 10^{12} \mathrm{M}_{\odot}$ (Fardal et al. 2013). Our MW mass is slightly less than half the total mass of the Local Group, $M_{\mathrm{LG}}=3.17 \pm 0.57 \times 10^{12} \mathrm{M}_{\odot}$ as derived by van der Marel et al. (2012), but slightly larger than half the estimate by Peñarrubia et al. (2016) of $M_{\mathrm{LG}}=$ $2.64_{-0.38}^{+0.42} \times 10^{12} \mathrm{M}_{\odot}$. Thus, the question of which galaxy is the most massive, between the MW and M31, remains open. For both galaxies there is the complication that there is a medium massive galaxy closeby (LMC for MW, M33 for M31) which is likely partly included in $M_{\text {vir }}$ estimates. On the one hand M33 is probably more massive, since it is more luminous (McConnachie 2012), on the other hand the LMC is closer to its host galaxy, and thus probably has a greater influence on mass estimates. Improved measurements may answer the question of which galaxy is the most massive in the Local Group.

While Gaia will improve its precision, the sample of satellites will maintain a bias towards those close to the Milky Way due to the rather bright detection limit. This is true for the Milky Way and even more so for M31: at its distance, all old stars are fainter than the detection limit of Gaia.

To obtain proper motions of more distant satellites other measurements are needed, in order to determine their 3-dimensional dynamics: these are in progress with HST (Kallivayalil et al. 2015; Kallivayalil 2016; Weisz et al. 2019).

\section{ACKNOWLEDGEMENTS}

The authors thank the referee for the thorough and constructive report, which significantly helped to improve the manuscript. TKF, GB, CB, and ST acknowledge financial support through the grants (AEI/FEDER, UE) AYA201789076-P, AYA2016-77237-C3-1-P and AYA2015-63810-P, as well as by the Ministerio de Ciencia, Innovación y Universidades (MCIU), through the State Budget and by the Consejer a de Economia, Industria, Comercio y Conocimiento of the Canary Islands Autonomous Community, through the Regional Budget. ADC acknowledges financial support from a Marie-Skłodowska-Curie Individual Fellowship grant, H2020-MSCA-IF-2016 Grant agreement 748213 DIGESTIVO. GB gratefully acknowledges financial support from Spanish Ministry of Economy and Competitiveness (MINECO) under the Ramon y Cajal Programme (RYC2012-11537). CB is supported by a MCIU Ramón y Cajal Fellowship (RYC 2013-12784). We thank Laura Watkins who provided data to test the mass estimator in the idealized case. We thank Mike Boylan-Kolchin for providing the $1 \sigma$ mass interval of Boylan-Kolchin et al. (2013) and Gwendolyn Eadie for providing the $1 \sigma$ mass intervals of Eadie \& Jurić (2019).

\section{REFERENCES}

Abbott T. M. C., et al., 2018, ApJS, 239, 18

Adén D., et al., 2009, A\&A, 506, 1147

Alves D. R., 2004, New Astron. Rev., 48, 659

Battaglia G., Starkenburg E., 2012, A\&A, 539, A123

Battaglia G., et al., 2005, MNRAS, 364, 433 
Bechtol K., et al., 2015, ApJ, 807, 50

Behroozi P., Wechsler R. H., Hearin A. P., Conroy C., 2019, MNRAS, 488, 3143

Bellazzini M., Ferraro F. R., Origlia L., Pancino E., Monaco L., Oliva E., 2002, AJ, 124, 3222

Bellazzini M., Gennari N., Ferraro F. R., 2005, MNRAS, 360, 185

Belokurov V., et al., 2007, ApJ, 654, 897

Binney J., Tremaine S., 2008, Galactic Dynamics: Second Edition. Princeton University Press

Bland-Hawthorn J., Gerhard O., 2016, ARA\&A, 54, 529

Boettcher E., et al., 2013, AJ, 146, 94

Bonanos A. Z., Stanek K. Z., Szentgyorgyi A. H., Sasselov D. D., Bakos G. Á., 2004, AJ, 127, 861

Bovy J., Bahmanyar A., Fritz T. K., Kallivayalil N., 2016, ApJ, 833,31

Boylan-Kolchin M., Springel V., White S. D. M., Jenkins A., 2010, MNRAS, 406, 896

Boylan-Kolchin M., Bullock J. S., Kaplinghat M., 2011, MNRAS, 415, L40

Boylan-Kolchin M., Bullock J. S., Sohn S. T., Besla G., van der Marel R. P., 2013, ApJ, 768, 140

Brook C. B., Di Cintio A., 2015, MNRAS, 450, 3920

Brook C. B., et al., 2012, MNRAS, 426, 690

Buchner J., et al., 2014, A\&A, 564, A125

Caldwell N., et al., 2017, ApJ, 839, 20

Callingham T. M., et al., 2019, MNRAS, 484, 5453

Carlin J. L., Sand D. J., 2018, ApJ, 865, 7

Carlin J. L., Grillmair C. J., Muñoz R. R., Nidever D. L., Majewski S. R., 2009, ApJ, 702, L9

Carlin J. L., et al., 2017, AJ, 154, 267

Carrera R., Aparicio A., Martínez-Delgado D., Alonso-García J., 2002, AJ, 123, 3199

Cautun M., et al., 2019, arXiv e-prints, p. arXiv:1911.04557

Chambers K. C., et al., 2016, preprint, (arXiv:1612.05560)

Cicuéndez L., et al., 2018, A\&A, 609, A53

Coppola G., et al., 2015, ApJ, 814, 71

D’Onghia E., Springel V., Hernquist L., Keres D., 2010, ApJ, 709, 1138

Dall'Ora M., et al., 2006, ApJ, 653, L109

Dall'Ora M., et al., 2012, ApJ, 752, 42

Deason A. J., Belokurov V., Evans N. W., An J., 2012, MNRAS, 424, L44

Deason A. J., Fattahi A., Belokurov V., Evans N. W., Grand R. J. J., Marinacci F., Pakmor R., 2019, MNRAS, 485, 3514

Dehnen W., McLaughlin D. E., Sachania J., 2006, MNRAS, 369,1688

Di Cintio A., Knebe A., Libeskind N. I., Hoffman Y., Yepes G., Gottlöber S., 2012, MNRAS, 423, 1883

Di Cintio A., Brook C. B., Dutton A. A., Macciò A. V., Stinson G. S., Knebe A., 2014, MNRAS, 441, 2986

Drlica-Wagner A., et al., 2015, ApJ, 813, 109

Drlica-Wagner A., et al., 2019, arXiv e-prints, p. arXiv:1912.03302

Eadie G., Jurić M., 2019, ApJ, 875, 159

Eadie G. M., Harris W. E., Widrow L. M., 2015, ApJ, 806, 54

Erkal D., et al., 2019, MNRAS, 487, 2685

Errani R., Peñarrubia J., 2019, arXiv e-prints, p. arXiv:1906.01642

Fardal M. A., et al., 2013, MNRAS, 434, 2779

Fardal M. A., van der Marel R. P., Law D. R., Sohn S. T., Sesar B., Hernitschek N., Rix H.-W., 2019, MNRAS, 483, 4724

Feroz F., Hobson M. P., Bridges M., 2009, MNRAS, 398, 1601

Fritz T. K., Battaglia G., Pawlowski M. S., Kallivayalil N., van der Marel R., Sohn S. T., Brook C., Besla G., 2018, A\&A, 619, A103

Fritz T. K., Carrera R., Battaglia G., Taibi S., 2019, A\&A, 623, A129

Gaia Collaboration et al., 2018a, A\&A, 616, A1
Gaia Collaboration et al., 2018b, A\&A, 616, A12

Garavito-Camargo N., Besla G., Laporte C. F. P., Johnston K. V., Gómez F. A., Watkins L. L., 2019, arXiv e-prints, p. arXiv:1902.05089

Garling C., et al., 2018, ApJ, 852, 44

Garofalo A., et al., 2013, ApJ, 767, 62

Garrison-Kimmel S., Boylan-Kolchin M., Bullock J. S., Lee K., 2014, MNRAS, 438, 2578

Garrison-Kimmel S., et al., 2018, MNRAS, 481, 4133

Gibbons S. L. J., Belokurov V., Evans N. W., 2014, MNRAS, 445,3788

Gnedin O. Y., Brown W. R., Geller M. J., Kenyon S. J., 2010, ApJ, 720, L108

Gómez F. A., Besla G., Carpintero D. D., Villalobos Á., O'Shea B. W., Bell E. F., 2015, ApJ, 802, 128

Graczyk D., et al., 2014, ApJ, 780, 59

Grand R. J. J., Deason A. J., White S. D. M., Simpson C. M., Gómez F. A., Marinacci F., Pakmor R., 2019, MNRAS, 487, L72

Gravity Collaboration et al., 2019, A\&A, 625, L10

Greco C., et al., 2008, ApJ, 675, L73

Grillmair C. J., 2009, ApJ, 693, 1118

Gullieuszik M., Held E. V., Rizzi L., Girardi L., Marigo P., Momany Y., 2008, MNRAS, 388, 1185

Hamanowicz A., et al., 2016, Acta Astron., 66, 197

Harris W. E., 1996, AJ, 112, 1487

Hattori K., Valluri M., Bell E. F., Roederer I. U., 2018, ApJ, 866,121

Huang Y., et al., 2016, MNRAS, 463, 2623

Hui L., Ostriker J. P., Tremaine S., Witten E., 2017, Phys. Rev. D, 95, 043541

Iocco F., Pato M., Bertone G., 2015, Nature Physics, 11, 245

Jethwa P., Erkal D., Belokurov V., 2016, MNRAS, 461, 2212

Johnston K. V., Zhao H., Spergel D. N., Hernquist L., 1999, ApJ, 512, L109

Joo S.-J., et al., 2018, ApJ, 861, 23

Kafle P. R., Sharma S., Lewis G. F., Bland-Hawthorn J., 2014, ApJ, 794, 59

Kafle P. R., Sharma S., Lewis G. F., Robotham A. S. G., Driver S. P., 2018, MNRAS, 475, 4043

Kallivayalil N., 2016, Milky Way Cosmology: Laying the Foundation for Full 6-D Dynamical Mapping of the Nearby Universe, HST Proposal

Kallivayalil N., et al., 2015, arXiv e-prints, p. arXiv:1503.01785

Kallivayalil N., et al., 2018, ApJ, 867, 19

Karukes E. V., Benito M., Iocco F., Trotta R., Geringer-Sameth A., 2019, arXiv e-prints, p. arXiv:1912.04296

Kelley T., Bullock J. S., Garrison-Kimmel S., Boylan-Kolchin M., Pawlowski M. S., Graus A. S., 2019, MNRAS, 487, 4409

Kim D., Jerjen H., 2015, ApJ, 808, L39

Kinemuchi K., Harris H. C., Smith H. A., Silbermann N. A., Snyder L. A., La Cluyzé A. P., Clark C. L., 2008, AJ, 136, 1921

Kirby E. N., Boylan-Kolchin M., Cohen J. G., Geha M., Bullock J. S., Kaplinghat M., 2013, ApJ, 770, 16

Kirby E. N., Simon J. D., Cohen J. G., 2015, ApJ, 810, 56

Kirby E. N., Cohen J. G., Simon J. D., Guhathakurta P., Thygesen A. O., Duggan G. E., 2017, ApJ, 838, 83

Koch A., Kleyna J. T., Wilkinson M. I., Grebel E. K., Gilmore G. F., Evans N. W., Wyse R. F. G., Harbeck D. R., 2007, AJ, 134,566

Koch A., et al., 2009, ApJ, 690, 453

Koposov S., et al., 2008, ApJ, 686, 279

Koposov S. E., et al., 2011, ApJ, 736, 146

Koposov S. E., Belokurov V., Torrealba G., Evans N. W., 2015a, ApJ, 805, 130

Koposov S. E., et al., 2015b, ApJ, 811, 62

Koposov S. E., et al., 2018, MNRAS, 479, 5343 
Kravtsov A. V., Vikhlinin A. A., Meshcheryakov A. V., 2018, Astronomy Letters, 44, 8

Kuehn C., et al., 2008, ApJ, 674, L81

Kulessa A. S., Lynden-Bell D., 1992, MNRAS, 255, 105

Küpper A. H. W., Balbinot E., Bonaca A., Johnston K. V., Hogg D. W., Kroupa P., Santiago B. X., 2015, ApJ, 803, 80

Laevens B. P. M., et al., 2014, ApJ, 786, L3

Li T. S., et al., 2018a, ApJ, 857, 145

Li T. S., et al., 2018b, ApJ, 866, 22

Li Z.-Z., Qian Y.-Z., Han J., Li T. S., Wang W., Jing Y. P., 2019, arXiv e-prints, p. arXiv:1912.02086

Liu L., Gerke B. F., Wechsler R. H., Behroozi P. S., Busha M. T., 2011, ApJ, 733, 62

Longeard N., et al., 2018, MNRAS, 480, 2609

Longeard N., et al., 2019, arXiv e-prints,

Martin N. F., Ibata R. A., Chapman S. C., Irwin M., Lewis G. F., 2007, MNRAS, 380, 281

Martin N. F., et al., 2016, MNRAS, 458, L59

Martínez-Vázquez C. E., et al., 2016, MNRAS, 462, 4349

Mateo M., Fischer P., Krzeminski W., 1995, AJ, 110, 2166

Mateo M., Olszewski E. W., Walker M. G., 2008, ApJ, 675, 201

McConnachie A. W., 2012, AJ, 144, 4

Medina G. E., et al., 2017, ApJ, 845, L10

Monari G., et al., 2018, A\&A, 616, L9

Moretti M. I., et al., 2009, ApJ, 699, L125

Musella I., et al., 2009, ApJ, 695, L83

Musella I., et al., 2012, ApJ, 756, 121

Mutlu-Pakdil B., et al., 2018, ApJ, 863, 25

Navarro J. F., Frenk C. S., White S. D. M., 1997, ApJ, 490, 493

Nidever D. L., et al., 2017, AJ, 154, 199

Pace A. B., Li T. S., 2019, ApJ, 875, 77

Pardy S. A., et al., 2019, arXiv e-prints,

Patel E., Besla G., Mandel K., Sohn S. T., 2018, ApJ, 857, 78

Pawlowski M. S., Pflamm-Altenburg J., Kroupa P., 2012, MNRAS, 423, 1109

Peñarrubia J., Gómez F. A., Besla G., Erkal D., Ma Y.-Z., 2016, MNRAS, 456, L54

Pietrzyński G., et al., 2008, AJ, 135, 1993

Piff T., et al., 2014, A\&A, 562, A91

Reid M. J., Brunthaler A., 2004, ApJ, 616, 872

Riley A. H., et al., 2019, MNRAS, 486, 2679

Rizzi L., Held E. V., Saviane I., Tully R. B., Gullieuszik M., 2007, MNRAS, 380, 1255

Rodríguez-Puebla A., Primack J. R., Avila-Reese V., Faber S. M., 2017, MNRAS, 470, 651

Sales L. V., Navarro J. F., Cooper A. P., White S. D. M., Frenk C. S., Helmi A., 2011, MNRAS, 418, 648

Samuel J., et al., 2019, arXiv e-prints, p. arXiv:1904.11508

Sand D. J., Strader J., Willman B., Zaritsky D., McLeod B., Caldwell N., Seth A., Olszewski E., 2012, ApJ, 756, 79

Schönrich R., Binney J., Dehnen W., 2010, MNRAS, 403, 1829

Schwarzschild M., 1979, ApJ, 232, 236

Shanks T., et al., 2015, MNRAS, 451, 4238

Simon J. D., 2018, ApJ, 863, 89

Simon J. D., Geha M., 2007, ApJ, 670, 313

Simon J. D., et al., 2011, ApJ, 733, 46

Simon J. D., et al., 2015, ApJ, 808, 95

Simon J. D., et al., 2017, ApJ, 838, 11

Sohn S. T., Watkins L. L., Fardal M. A., van der Marel R. P., Deason A. J., Besla G., Bellini A., 2018, ApJ, 862, 52

Somerville R. S., et al., 2018, MNRAS, 473, 2714

Spencer M. E., Mateo M., Walker M. G., Olszewski E. W., McConnachie A. W., Kirby E. N., Koch A., 2017, AJ, 153, 254

Stetson P. B., Fiorentino G., Bono G., Bernard E. J., Monelli M., Iannicola G., Gallart C., Ferraro I., 2014, PASP, 126, 616

Torrealba G., et al., 2016, MNRAS, 463, 712

Torrealba G., et al., 2018, MNRAS, 475, 5085

Torrealba G., et al., 2019, MNRAS, p. 1548
Vasiliev E., 2019, MNRAS, 484, 2832

Vera-Ciro C. A., Helmi A., Starkenburg E., Breddels M. A., 2013, MNRAS, 428, 1696

Vivas A. K., Mateo M., 2013, AJ, 146, 141

Vivas A. K., et al., 2016, AJ, 151, 118

Voggel K., Hilker M., Baumgardt H., Collins M. L. M., Grebel E. K., Husemann B., Richtler T., Frank M. J., 2016, MNRAS, 460, 3384

Walker M. G., Belokurov V., Evans N. W., Irwin M. J., Mateo M., Olszewski E. W., Gilmore G., 2009a, ApJ, 694, L144

Walker M. G., Mateo M., Olszewski E. W., Peñarrubia J., Wyn Evans N., Gilmore G., 2009b, ApJ, 704, 1274

Walker M. G., Olszewski E. W., Mateo M., 2015a, MNRAS, 448, 2717

Walker M. G., Olszewski E. W., Mateo M., 2015b, MNRAS, 448, 2717

Walker M. G., et al., 2016, ApJ, 819, 53

Walsh S. M., Willman B., Sand D., Harris J., Seth A., Zaritsky D., Jerjen H., 2008, ApJ, 688, 245

Wang J., Frenk C. S., Navarro J. F., Gao L., Sawala T., 2012, MNRAS, 424, 2715

Wang W., Han J., Cooper A. P., Cole S., Frenk C., Lowing B., 2015a, MNRAS, 453, 377

Wang L., Dutton A. A., Stinson G. S., Macciò A. V., Penzo C., Kang X., Keller B. W., Wadsley J., 2015b, MNRAS, 454, 83

Wang W., Han J., Cautun M., Li Z., Ishigaki M. N., 2019, arXiv e-prints, p. arXiv:1912.02599

Watkins L. L., Evans N. W., An J. H., 2010, MNRAS, 406, 264

Watkins L. L., van der Marel R. P., Sohn S. T., Evans N. W., 2019, ApJ, 873, 118

Wechsler R. H., Tinker J. L., 2018, ARA\&A, 56, 435

Weisz D. R., et al., 2016, ApJ, 822, 32

Weisz D. R., et al., 2019, Tracing the 6-D Orbital and Formation History of the Complete M31 Satellite System, HST Proposal

Wetzel A. R., Hopkins P. F., Kim J.-h., Faucher-Giguère C.-A., Kereš D., Quataert E., 2016, ApJ, 827, L23

White S. D. M., Rees M. J., 1978, MNRAS, 183, 341

Willman B., et al., 2006, arXiv e-prints, pp astro-ph/0603486

Xue X. X., et al., 2008, ApJ, 684, 1143

York D. G., et al., 2000, AJ, 120, 1579

di Cintio A., Knebe A., Libeskind N. I., Yepes G., Gottlöber S., Hoffman Y., 2011, MNRAS, 417, L74

van den Bosch F. C., Ogiya G., Hahn O., Burkert A., 2018, MNRAS, 474, 3043

van der Marel R. P., Fardal M., Besla G., Beaton R. L., Sohn S. T., Anderson J., Brown T., Guhathakurta P., 2012, ApJ, 753,8

\section{APPENDIX A: DETAILS ON SAMPLE}

We list in Table A1 the sources of the used properties like distance modulus, proper motion and line-of-sight velocity. For further details, see Fritz et al. (2018).

This paper has been typeset from a $\mathrm{T}_{\mathrm{EX}} \mathrm{LATEX}$ file prepared by the author. 
Table A1. Sample of galaxies. Col. 1 lists the object name; in col. 2 we provide the distance moduli (d.m.) used and their source; in col. 3 we provide the line of sight velocities and their source ; in col. $4 / 5$ we list the proper motions and their sources in both dimension and in col. 6 the systematic uncertainties of both.

\begin{tabular}{|c|c|c|c|c|c|}
\hline name & d.m. & $\mathrm{v}_{\text {l.o.s. }}$ & $\mu_{\alpha}$ & $\mu_{\delta}$ & syst. $\mu$ uncertainty \\
\hline & & $\mathrm{km} / \mathrm{s}$ & [mas/yr] & $\overline{[\mathrm{mas} / \mathrm{yr}]}$ & [mas/yr] \\
\hline Antila II & $20.60 \pm 0.11^{77}$ & $291^{77}$ & $-0.095 \pm 0.187^{77}$ & $0.058 \pm 0.024^{77}$ & $0.035^{\text {This paper }}$ \\
\hline Aquarius II & $20.16 \pm 0.07^{1}$ & $-71^{1}$ & $-0.252 \pm 0.526^{72}$ & $0.011 \pm 0.448^{72}$ & $0.063^{72}$ \\
\hline Boötes I & $19.11 \pm 0.08^{32}$ & $99^{2,30}$ & $-0.554 \pm 0.092^{72}$ & $-1.111 \pm 0.068^{72}$ & $0.035^{72}$ \\
\hline Boötes II & $18.11 \pm 0.06^{33}$ & $-117^{3}$ & $-2.686 \pm 0.389^{72}$ & $-0.53 \pm 0.287^{72}$ & $0.056^{72}$ \\
\hline Boötes III & $18.35 \pm 0.1^{74}$ & $198^{73}$ & $-1.14 \pm 0.18^{75}$ & $-0.98 \pm 0.2^{75}$ & $0.035^{\text {This }}$ paper \\
\hline Canes Venatici I & $21.62 \pm 0.05^{34}$ & $31^{2,4}$ & $-0.159 \pm 0.094^{72}$ & $-0.067 \pm 0.054^{72}$ & $0.035^{72}$ \\
\hline Canes Venatici II & $21.02 \pm 0.06^{35}$ & $-129^{4}$ & $-0.342 \pm 0.232^{72}$ & $-0.473 \pm 0.169^{72}$ & $0.056^{72}$ \\
\hline Carina I & $20.08 \pm 0.08^{57,58}$ & $229^{5}$ & $0.485 \pm 0.017^{72}$ & $0.131 \pm 0.016^{72}$ & $0.035^{72}$ \\
\hline Carina II & $17.79 \pm 0.05^{36}$ & $477^{6}$ & $1.867 \pm 0.078^{72}$ & $0.082 \pm 0.072^{72}$ & $0.035^{72}$ \\
\hline Carina III & $17.22 \pm 0.10^{36}$ & $285^{6}$ & $3.046 \pm 0.119^{72}$ & $1.565 \pm 0.135^{72}$ & $0.057^{72}$ \\
\hline Columba II & $21.31 \pm 0.11^{53}$ & $156^{76}$ & $0.33 \pm 0.28^{76}$ & $-0.38 \pm 0.38^{76}$ & $0.062^{76}$ \\
\hline Coma Berenices I & $18.13 \pm 0.08^{37}$ & $98^{4}$ & $0.471 \pm 0.108^{72}$ & $-1.716 \pm 0.104^{72}$ & $0.035^{72}$ \\
\hline Crater II & $20.25 \pm 0.10^{39}$ & $88^{9}$ & $-0.184 \pm 0.061^{72}$ & $-0.106 \pm 0.031^{72}$ & $0.035^{72}$ \\
\hline Draco I & $19.49 \pm 0.17^{59,60}$ & $-291^{10}$ & $-0.012 \pm 0.013^{72}$ & $-0.158 \pm 0.015^{72}$ & $0.035^{72}$ \\
\hline Draco II & $16.66 \pm 0.04^{40}$ & $-348^{11}$ & $1.242 \pm 0.276^{72}$ & $0.845 \pm 0.285^{72}$ & $0.057^{72}$ \\
\hline Fornax I & $20.72 \pm 0.04^{61}$ & $55^{5,13}$ & $0.374 \pm 0.004^{72}$ & $-0.401 \pm 0.005^{72}$ & $0.035^{72}$ \\
\hline Grus I & $20.4 \pm 0.2^{44}$ & $-141^{14}$ & $-0.261 \pm 0.172^{72}$ & $-0.437 \pm 0.238^{72}$ & $0.046^{72}$ \\
\hline Hercules I & $20.64 \pm 0.14^{42,43}$ & $45^{4,15}$ & $-0.297 \pm 0.118^{72}$ & $-0.329 \pm 0.094^{72}$ & $0.035^{72}$ \\
\hline Horologium I & $19.46 \pm 0.2^{44,45}$ & $169^{16}$ & $1.52 \pm 0.25^{72}$ & $-0.47 \pm 0.39^{72}$ & $0.058^{72}$ \\
\hline Horologium II & $19.6 \pm 0.2^{78}$ & $113^{76}$ & $0.891 \pm 0.088^{76}$ & $-0.55 \pm 0.08^{76}$ & $0.049^{76}$ \\
\hline Hydra II & $20.89 \pm 0.12^{46}$ & $303^{7}$ & $-0.416 \pm 0.519^{72}$ & $0.134 \pm 0.422^{72}$ & $0.061^{72}$ \\
\hline Hydrus I & $17.20 \pm 0.04^{29}$ & $80^{29}$ & $4.044 \pm 0.312^{72}$ & $-1.755 \pm 0.276^{72}$ & $0.035^{72}$ \\
\hline Leo I & $22.15 \pm 0.1^{62}$ & $283^{17}$ & $-0.086 \pm 0.059^{72}$ & $-0.128 \pm 0.062^{72}$ & $0.035^{72}$ \\
\hline Leo II & $21.76 \pm 0.13^{63,64}$ & $78^{18,31}$ & $-0.025 \pm 0.08^{72}$ & $-0.173 \pm 0.083^{72}$ & $0.035^{72}$ \\
\hline Leo IV & $20.94 \pm 0.07^{47}$ & $132^{4}$ & $-0.59 \pm 0.531^{72}$ & $-0.449 \pm 0.358^{72}$ & $0.059^{72}$ \\
\hline Leo V & $21.19 \pm 0.06^{48}$ & $173^{19}$ & $-0.097 \pm 0.557^{72}$ & $-0.628 \pm 0.302^{72}$ & $0.057^{72}$ \\
\hline LMC & $18.50 \pm 0.02^{80}$ & $262^{83}$ & $1.85^{82}$ & $0.234^{82}$ & $0.030^{82}$ \\
\hline SMC & $18.99 \pm 0.12^{80}$ & $146^{80,84}$ & $0.797^{82}$ & $-1.22^{82}$ & $0.030^{82}$ \\
\hline Pisces II & $21.31 \pm 0.18^{49}$ & $-227^{7}$ & $-0.108 \pm 0.645^{72}$ & $-0.586 \pm 0.498^{72}$ & $0.061^{72}$ \\
\hline Phoenix II & $19.60 \pm 0.1^{50}$ & $33^{76}$ & $0.5 \pm 0.12^{76}$ & $-1.16 \pm 0.14^{76}$ & $0.059^{76}$ \\
\hline Reticulum II & $17.5 \pm 0.1^{50}$ & $63^{21}$ & $2.398 \pm 0.04^{72}$ & $-1.319 \pm 0.048^{72}$ & $0.035^{72}$ \\
\hline Reticulum III & $19.82 \pm 0.31^{79}$ & $274^{76}$ & $-0.39 \pm 0.53^{76}$ & $-0.32 \pm 0.63^{76}$ & $0.058^{76}$ \\
\hline Sagittarius I & $17.13 \pm 0.11^{65}$ & $140^{80}$ & $-2.736 \pm 0.009^{72}$ & $-1.357 \pm 0.008^{72}$ & $0.035^{72}$ \\
\hline Sagittarius II & $19.32 \pm 0.03^{81}$ & $-177^{81}$ & $-0.65 \pm 0.09^{81}$ & $-0.88 \pm 0.12^{81}$ & $0.035^{85}$ \\
\hline Sculptor I & $19.64 \pm 0.13^{67,68}$ & $111^{5,13}$ & $0.084 \pm 0.006^{72}$ & $-0.133 \pm 0.006^{72}$ & $0.035^{72}$ \\
\hline Segue 1 & $16.8 \pm 0.2^{51}$ & $209^{22}$ & $-1.697 \pm 0.195^{72}$ & $-3.501 \pm 0.175^{72}$ & $0.035^{72}$ \\
\hline Segue 2 & $17.8 \pm 0.18^{52}$ & $-39^{23}$ & $1.656 \pm 0.155^{72}$ & $0.135 \pm 0.104^{72}$ & $0.045^{72}$ \\
\hline Sextans I & $19.67 \pm 0.15^{68}$ & $224^{24}$ & $-0.438 \pm 0.028^{72}$ & $0.055 \pm 0.028^{72}$ & $0.035^{72}$ \\
\hline Triangulum II & $17.27 \pm 0.1^{53}$ & $-382^{25}$ & $0.588 \pm 0.187^{72}$ & $0.554 \pm 0.161^{72}$ & $0.051^{72}$ \\
\hline Tucana II & $18.8 \pm 0.2^{44,45}$ & $-129^{14}$ & $0.91 \pm 0.059^{72}$ & $-1.159 \pm 0.074^{72}$ & $0.035^{72}$ \\
\hline Tucana III & $16.8 \pm 0.1^{50}$ & $-102^{26,27}$ & $-0.025 \pm 0.034^{72}$ & $-1.661 \pm 0.035^{72}$ & $0.035^{72}$ \\
\hline Ursa Major I & $19.94 \pm 0.13^{54}$ & $-55^{2,4}$ & $-0.683 \pm 0.094^{72}$ & $-0.72 \pm 0.13^{72}$ & $0.035^{72}$ \\
\hline Ursa Major II & $17.70 \pm 0.13^{55}$ & $-117^{2,4}$ & $1.691 \pm 0.053^{72}$ & $-1.902 \pm 0.066^{72}$ & $0.035^{72}$ \\
\hline Ursa Minor I & $19.40 \pm 0.11^{70,71}$ & $-247^{28}$ & $-0.184 \pm 0.026^{72}$ & $0.082 \pm 0.023^{72}$ & $0.035^{72}$ \\
\hline Willman 1 & $17.90 \pm 0.40^{56}$ & $-12^{2}$ & $0.199 \pm 0.187^{72}$ & $-1.342 \pm 0.366^{72}$ & $0.051^{72}$ \\
\hline
\end{tabular}

(1) Torrealba et al. (2016); (2) Martin et al. (2007); (3) Koch et al. (2009); (4) Simon \& Geha (2007); (5) Walker et al. (2015a); (6) Li et al. (2018a); (7) Kirby et al. (2015); (9) Caldwell et al. (2017); (10) Walker et al. (2015b); (11) Martin et al. (2016); (13) Battaglia \& Starkenburg (2012, and references therein); (14) Walker et al. (2016); (15) Adén et al. (2009); (16) Koposov et al. (2015b); (17) Mateo et al. (2008); (18) Spencer et al. (2017); (19) Walker et al. (2009a); (21) Simon et al. (2015); (22) Simon et al. (2011); (23) Kirby et al. (2013); (24) Cicuéndez et al. (2018); (25) Kirby et al. (2017); (26) Simon et al. (2017); (27) Li et al. (2018b); (28)

Walker et al. (2009b), (29) Koposov et al. (2018); (30) Koposov et al. (2011); (31) Koch et al. (2007); (32) Dall'Ora et al. (2006); (33) Walsh et al. (2008); (34) Kuehn et al. (2008); (35) Greco et al. (2008); (36) Torrealba et al. (2018); (37) Musella et al. (2009); (38)

Weisz et al. (2016); (39) Joo et al. (2018); (40) Longeard et al. (2018); (42) Musella et al. (2012); (43) Garling et al. (2018); (44) Koposov et al. (2015a); (45) Bechtol et al. (2015); (46)Vivas et al. (2016); (47) Moretti et al. (2009); (48) Medina et al. (2017); (49) Sand et al. (2012); (50) Mutlu-Pakdil et al. (2018); (51) Belokurov et al. (2007); (52) Boettcher et al. (2013); (53) Carlin et al. (2017);

(54) Garofalo et al. (2013); (55) Dall'Ora et al. (2012); (56) Willman et al. (2006), (57) Coppola et al. (2015); (58) Vivas \& Mateo (2013); (59) Bonanos et al. (2004); (60) Kinemuchi et al. (2008); (61) Rizzi et al. (2007); (62) Stetson et al. (2014); (63)

Bellazzini et al. (2005); (64) Gullieuszik et al. (2008); (65) Hamanowicz et al. (2016); (67) Martínez-Vázquez et al. (2016); (68)

Pietrzyński et al. (2008); (69) Mateo et al. (1995); (70) Carrera et al. (2002); (71) Bellazzini et al. (2002); (72) Fritz et al. (2018); (73)

Carlin et al. (2009); (74) Grillmair (2009); (75) Carlin \& Sand (2018); (76) Fritz et al. (2019); (77) Torrealba et al. (2019); (78)

Kim \& Jerjen (2015); (79) Drlica-Wagner et al. (2015); (80) McConnachie (2012); (81) Longeard et al. (2019); (82)

MNRAS 000, 1-16 (2020) Gaia Collaboration et al. (2018b); (83) Alves (2004); (84) Graczyk et al. (2014). 